\title{
THE GROUND STATES OF LARGE QUANTUM DOTS IN MAGNETIC FIELDS ${ }^{\dagger}$
}

\author{
Elliott H. Lieb* and Jan Philip Solovej** \\ Department of Mathematics, Fine Hall, Princeton University, Princeton, NJ 08544 \\ Jakob Yngvason*** \\ Science Institute, University of Iceland, Dunhaga 3, IS-107 Reykjavik, Iceland
}

\begin{abstract}
The quantum mechanical ground state of a 2D $N$-electron system in a confining potential $V(x)=K v(x)$ ( $K$ is a coupling constant) and a homogeneous magnetic field $B$ is studied in the high density limit $N \rightarrow \infty, K \rightarrow \infty$ with $K / N$ fixed. It is proved that the ground state energy and electronic density can be computed exactly in this limit by minimizing simple functionals of the density. There are three such functionals depending on the way $B / N$ varies as $N \rightarrow \infty:$ A $2 \mathrm{D}$ Thomas-Fermi (TF) theory applies in the case $B / N \rightarrow 0$; if $B / N \rightarrow$ const. $\neq 0$ the correct limit theory is a modified $B$-dependent TF model, and the case $B / N \rightarrow \infty$ is described by a "classical" continuum electrostatic theory. For homogeneous potentials this last model describes also the weak coupling limit $K / N \rightarrow 0$ for arbitrary $B$. Important steps in the proof are the derivation of a new Lieb-Thirring inequality for the sum of eigenvalues of single particle Hamiltonians in 2D with magnetic fields, and an estimation of the exchange-correlation energy. For this last estimate we study a model of classical point charges with electrostatic interactions that provides a lower bound for the true quantum mechanical energy.
\end{abstract}

PACS numbers: 73.20.Dx, 31.20.Lr, 71.10+x, 71.45Jp, 03.65.Db, 03.65.Sq

\footnotetext{
$\dagger$ To appear in Phys. Rev. B

* Work partially supported by U.S. National Science Foundation grant PHY90-19433 A03

** Work partially supported by U.S. National Science Foundation grant DMS 92-03829

*** Work partially supported by the Icelandic Science Foundation and the Research Fund of the University of Iceland.
} 


\section{INTRODUCTION}

In the last few years considerable experimental and theoretical work has been devoted to the study of quantum dots, which are atomic-like two-dimensional systems, confined within semiconductor heterostructures. ${ }^{*}$ The parameters of such artificial atoms may differ appreciately from their natural counterparts because of the interactions of the electrons with the crystal where they reside. In a quantum dot the natural atomic unit of length is $a_{*}=\epsilon \hbar^{2} /\left(m_{*} e^{2}\right)$, where $\epsilon$ is the dielectric constant and $m_{*}$ is the effective electron mass. Compared with the usual Bohr radius, $a_{0}=\hbar^{2} /\left(m e^{2}\right)$, the length $a_{*}$ is typically large, e.g., $a_{*} \approx 185 a_{0}$ in GaAs. The corresponding natural unit, $B_{*}$, with which we measure the magnetic field, $B$, is the field at which the magnetic length $\ell_{B}=\hbar e /\left(B^{1 / 2} c\right)$ equals $a_{*}$, i.e., $B_{*}=\left(a_{0} / a_{*}\right)^{2} B_{0}$, where $B_{0}=e^{3} m^{2} c / \hbar^{3}=2.35 \times 10^{5} \mathrm{~T}$ is the value corresponding to free electrons. If $a_{0} / a_{*}$ is small, $B_{*}$ can be much smaller than $B_{0}$. Thus $B_{*} \approx 7 \mathrm{~T}$ in GaAs. This makes it possible to study in the laboratory effects which, for natural atoms, require the magnetic fields of white dwarfs or even neutron stars.

The ground state properties of natural atoms in high magnetic fields have recently been analyzed rigorously in the asymptotic limit where the number of electrons and the nuclear charge are large [19-21]. For artificial atoms one may expect asymptotic analysis to be even more useful because the accuracy increases with the number of electrons, and a quantum dot can easily accommodate several hundred or even a thousand electrons. In the present paper we carry out such an analysis of the ground state of a quantum dot in a magnetic field. One of our conclusions is that the "self consistent model" introduced by McEuen et al $[3,13]$ is a rigorous limit of quantum mechanics. This model has recently

\footnotetext{
* The number of articles on this subject is by now quite large. See, e.g., [1], [2] for reviews,
} [3]-[7] for recent measurements of conductivity and capacity of quantum dots, and [8]-[18] for various theoretical aspects and further references. 
been applied to explain interesting features of the addition spectra of large quantum dots in strong magnetic fields $[15,6]$.

Before discussing our results for dots we summarize, for comparison, the main findings about atoms in [19-21]. The quantum mechanical ground state energy and electronic density of a natural atom or ion with electron number $N$ and nuclear charge $Z$ in a homogeneous magnetic field $B$ can, in the limit $N \rightarrow \infty, Z \rightarrow \infty$ with $Z / N$ fixed, be described exactly by functionals of the density, or, in one case, of density matrices. There are five different functionals, depending on the way $B$ varies with $N$ as $N \rightarrow \infty$. In each of the cases $B \ll N^{4 / 3}$ (with $B$ measured in the natural unit $B_{0}$ ), $B \sim N^{4 / 3}$ and $N^{4 / 3} \ll B \ll N^{3}$ the correct asymptotics is given by an appropriate functional of the semiclassical Thomas-Fermi type. For $B \sim N^{3}$ a novel type of functional, depending on density matrices is required, whereas the case $B \gg N^{3}$ is described by a density functional that can be minimized in closed form.

A review of our results about quantum dots was given in [22]. Due to the reduced dimensionality of the electronic motion, there are only three different asymptotic theories for quantum dots instead of five for natural atoms. These three theories are given by simple functionals of the density and correspond respectively to the cases $B \ll N, B \sim N$ and $B \gg N\left(B\right.$ measured in units of $\left.B_{*}\right)$ as $N \rightarrow \infty$ with $V / N$ fixed, where $V$ is the attractive exterior potential that restricts the two-dimensional motion of the electrons. This potential, which plays the same role as the nuclear attraction in a natural atom, is generated in a quantum dot by exterior gates, and thus is adjustable to a certain extent. In the course of proving the asymptotic limits we shall also consider, in addition to the density functionals, a model of classical point charges in two dimension that gives a lower bound to the quantum-mechanical energy. 
Some of the methods and results of the present paper contrast markedly with those of our earlier work [19-21]. From a mathematical point of view the most interesting feature of quantum dots compared to natural atoms is the somewhat peculiar electrostatics that appears because the interaction between the electrons is given by the three dimensional Coulomb potential although the motion is two dimensional. Also, the fact that the kinetic energy vanishes in the lowest Landau level requires additional mathematical effort in order to bound the kinetic energy from below by a functional of the density. We now describe in more detail the limit theorems to be proved in the sequel. A quantum dot with $N$ electrons in a confining potential $V$ and a homogeneous magnetic field $B$ is modeled by the following Hamiltonian:

$$
H_{N}=\sum_{j=1}^{N} H_{1}^{(j)}+\frac{e^{2}}{\epsilon} \sum_{1 \leq i<j \leq N}\left|x_{i}-x_{j}\right|^{-1}
$$

with $x_{i} \in \mathbf{R}^{2}$ and where $H_{1}$ is the one-body Hamiltonian

$$
H_{1}=\frac{\hbar^{2}}{2 m_{*}}\left(i \nabla-\frac{e}{\hbar c} \mathbf{A}\right)^{2}+g_{*}\left(\frac{\hbar e}{2 m c}\right) \mathbf{S} \cdot \mathbf{B}-\left(\frac{\hbar e}{2 m c}\right)\left(\frac{m}{m_{*}}-\frac{\left|g_{*}\right|}{2}\right) B+V(x)
$$

As before, $e$ and $m$ denote the charge and mass of a (free) electron, $\epsilon$ is the dielectric constant, $m_{*}$ is the effective mass, and $g_{*}$ is the effective $g$-factor. The magnetic vector potential is $\mathbf{A}(x)=\frac{1}{2}\left(-B x^{1}, B x^{2}\right)$ (with $\left.x=\left(x^{1}, x^{2}\right) \in \mathbf{R}^{2}\right), \mathbf{B}=(0,0, B)$ and $\mathbf{S}$ is the vector of electron spin operators. The potential $V(x)$ is supposed to be continuous and confining, which is to say that $V(x) \rightarrow \infty$ as $|x| \rightarrow \infty$. It is not assumed to be circularly symmetric. The constant term in $(1.2),-(\hbar e /(2 m c))\left(\left(m / m_{*}\right)-\left|g_{*}\right| / 2\right) B$, is included in order that the "kinetic energy" operator, $H_{\text {kin }}=H_{1}-V(x)$, has a spectrum starting at zero. The Hilbert space is that appropriate for fermions with spin, the antisymmetric tensor product $\bigwedge_{1}^{N} L^{2}\left(\mathbf{R}^{2} ; \mathbf{C}^{2}\right)$. 
We define an effective charge by $e_{*}=e / \sqrt{\epsilon}$ and choose units such that $\hbar=m_{*}=$ $e_{*}=1$. The unit of length is then the effective Bohr radius $a_{*}=\hbar^{2} /\left(m_{*} e_{*}^{2}\right)$ and the unit of energy is $E_{*}=e_{*}^{2} / a_{*}=e_{*}^{4} m_{*} / \hbar^{2}$. Moreover, the unit $B_{*}$ for the magnetic field is determined by $\hbar e B_{*} /\left(m_{*} c\right)=E_{*}$, so $B_{*}=e_{*}^{3} m_{*}^{2} c /\left(\epsilon^{1 / 2} \hbar^{3}\right)$. The values for GaAs are $a_{*}=9.8 \mathrm{~nm}, E_{*}=12 \mathrm{meV}$ and $B_{*}=6.7 \mathrm{~T}$.

The true quantum-mechanical ground state energy of $H_{N}$ is denoted by $E^{\mathrm{Q}}(N, B, V)$ and the true ground state electron density by $\rho_{N, B, V}^{\mathrm{Q}}(x)$. The density functionals that describe the asymptotics of $E^{\mathrm{Q}}$ and $\rho^{\mathrm{Q}}$ are of three types. The first is a standard twodimensional Thomas-Fermi energy functional

$$
\mathcal{E}^{\mathrm{TF}}[\rho ; V]=(\pi / 2) \int \rho(x)^{2} \mathrm{~d} x+\int V(x) \rho(x) \mathrm{d} x+D(\rho, \rho)
$$

with

$$
D(\rho, \rho)=\frac{1}{2} \iint \frac{\rho(x) \rho(y)}{|x-y|} \mathrm{d} x \mathrm{~d} y
$$

Here $\rho$ is a nonnegative density on $\mathbf{R}^{2}$ and all integrals are over $\mathbf{R}^{2}$ unless otherwise stated. The second functional is a two-dimensional "magnetic Thomas Fermi functional"

$$
\mathcal{E}^{\mathrm{MTF}}[\rho ; B, V]=\int j_{B}(\rho(x)) \mathrm{d} x+\int V(x) \rho(x) \mathrm{d} x+D(\rho, \rho)
$$

where $j_{B}$ is a piece-wise linear function that will be defined precisely in the next section. This functional is the two dimensional analogue of the three dimensional magnetic Thomas Fermi functional that was introduced in [23] and further studied in [24,25,21]. The present two dimensional version was first stated in [3]; these authors call it the self-consistent model $(\mathrm{SC})^{*}$.

* The repulsion term considered in [3] is slightly different from $D(\rho, \rho)$, since it has cut-offs at long and short distances. It is still positive definite as a kernel and our methods can easily be adapted to prove Theorems 1.1 and 1.2 with such cut-off Coulomb kernels. 
The last asymptotic functional will be called the "classical functional", since the kinetic energy term is absent and only classical interactions remain:

$$
\mathcal{E}^{\mathrm{C}}[\rho ; V]=\int V(x) \rho(x) \mathrm{d} x+D(\rho, \rho) .
$$

The functionals (1.3) and (1.6) are in fact limiting cases of (1.5) for $B \rightarrow 0$ and $B \rightarrow \infty$ respectively. As discussed in detail later, for each functional there is a unique density that minimizes it under the constraint $\int \rho=N$. We denote these densities respectively by $\rho_{N, V}^{\mathrm{TF}}(x), \rho_{N, B, V}^{\mathrm{MTF}}(x), \rho_{N, V}^{\mathrm{C}}(x)$, and the corresponding minimal energies by $E^{\mathrm{TF}}(N, V)$, $E^{\mathrm{MTF}}(N, B, V)$ and $E^{\mathrm{C}}(N, V)$.

In order to relate $E^{\mathrm{Q}}$ to these other energies we take a high density limit. This is achieved by letting $N$ tend to infinity (which is a reasonable thing to do physically, since $N$ can be several hundred) and we let $V$ tend to infinity. The latter statement means that we fix a potential $v$ and set $V=N v$. With this understanding of $N, V \rightarrow \infty$ our main results are summarized in the following two theorems. [In order to prove these theorems we need to assume that $V$ is sufficiently regular. The technical requirement is that $V$ belongs to the class $C_{\text {loc }}^{1, \alpha}$, (see Theorem 3.2 for the definition of $\left.\left.C_{\mathrm{loc}}^{1, \alpha}\right)\right]$.

1.1 THEOREM (Limit theorem for the energy). Let $V=N v$ with $v$ a fixed function in $C_{\mathrm{loc}}^{1, \alpha}$. Then

$$
\lim _{N \rightarrow \infty} E^{\mathrm{Q}}(N, B, V) / E^{\mathrm{MTF}}(N, B, V)=1
$$

uniformly in B. Moreover,

$$
\lim _{N \rightarrow \infty} E^{\mathrm{Q}}(N, B, V) / E^{\mathrm{TF}}(N, V)=1 \quad \text { if } B / N \rightarrow 0
$$

and

$$
\lim _{N \rightarrow \infty} E^{\mathrm{Q}}(N, B, V) / E^{\mathrm{C}}(N, V)=1 \quad \text { if } B / N \rightarrow \infty .
$$


1.2 THEOREM (Limit theorem for the density). Let $V=N v$ with $v$ a fixed function in $C_{\mathrm{loc}}^{1, \alpha}$. Then

$$
\frac{1}{N} \rho_{N, B, V}^{\mathrm{Q}} \rightarrow \rho_{1, B / N, v}^{\mathrm{MTF}}
$$

uniformly in $B$, and

$$
\begin{aligned}
& \frac{1}{N} \rho_{N, B, V}^{\mathrm{Q}} \rightarrow \rho_{1, v}^{\mathrm{TF}} \quad \text { if } B / N \rightarrow 0, \\
& \frac{1}{N} \rho_{N, B, V}^{\mathrm{Q}} \rightarrow \rho_{1, v}^{\mathrm{C}} \quad \text { if } B / N \rightarrow \infty .
\end{aligned}
$$

The convergence is in the weak $L^{1}$ sense*

Let us add a few comments on these results. As discussed in the Section II, the energy $E^{\mathrm{MTF}}$ has the scaling property

$$
E^{\mathrm{MTF}}(N, B, V)=N^{2} E^{\mathrm{MTF}}(1, B / N, V / N)
$$

Thus (1.7) is equivalent to

$$
E^{\mathrm{Q}}(N, V, B)=N^{2} E^{\mathrm{MTF}}(1, B / N, V / N)+o\left(N^{2}\right)
$$

where the error term is uniformly bounded in $B$ for $V / N$ fixed. One expects the error to be $O\left(N^{3 / 2}\right)$, which is the order of the exchange contribution to the Coulomb interaction, but our methods do not quite allow us to prove this. We do, however, show that for $B / N$ larger than a critical value (depending on $V / N)$ one has $E^{\mathrm{MTF}}(N, B, V)=E^{\mathrm{C}}(N, V)$ and

$$
E^{\mathrm{Q}}(N, V, B) \geq N^{2} E^{\mathrm{C}}(1, V / N)-b N^{3 / 2}
$$

where the coefficient $b$ depends only on $V / N$.

* By definition, a sequence of functions $f_{n}$ converges to a function $f$ in weak $L^{1}$ sense if $\int f_{n} g \rightarrow \int f g$ for all bounded (measurable) functions $g$. 
The condition that $V / N$ is fixed as $N \rightarrow \infty$ guarantees that the diameter of the electronic density distribution stays bounded as $N \rightarrow \infty$; thus the limit we are considering is really a high density limit rather than simply a large $N$ limit. On the other hand, for a homogeneous potential $V$ (e.g., quadratic, as is often assumed) one obtains also a nontrivial $N \rightarrow \infty$ limit for $V$ fixed, if the lengths are suitably scaled. In fact, this limit is given by the classical functional (1.6). Intuitively this is easy to understand, for if an increase in $N$ is not compensated by an increase in $V$ the charge density spreads out and the kinetic energy terms in (1.5) and (1.3) become negligible compared with the other terms. (The result again requires $V$ to be in $C_{\mathrm{loc}}^{1, \alpha}$.)

1.3 THEOREM (Energy limit with a homogeneous potential). Assume that $v$ is homogeneous of degree $s \geq 1$, i.e.,

$$
v(\lambda x)=\lambda^{s} v(x) .
$$

Then

$$
\lim _{N \rightarrow \infty} E^{\mathrm{Q}}(N, B, K v) / E^{\mathrm{MTF}}(N, B, K v)=1
$$

uniformly in $B$ and in $K$ as long as $K / N$ is bounded above. Moreover, if $K / N \rightarrow 0$ as $N \rightarrow \infty$, then

$$
\lim _{N \rightarrow \infty} E^{\mathrm{Q}}(N, B, K v) / E^{\mathrm{C}}(N, B)=1
$$

uniformly in $B$.

One can also prove a limit theorem for the density in the case of homogeneous potentials. Since the formulation of such a theorem becomes somewhat complicated we refrain from doing this, but refer to Eqs. (2.14)-(2.16) below for the scaling of the MTF functional with $k=K / N$ and to (3.24) for the weak coupling limit of the MTF density.

The proof of the limit theorems involves the following steps. In sections II and III we discuss the basic properties of the functionals (1.3)-(1.6). In Section IV we consider 
the energy of a system of classical point charged particles in $\mathbf{R}^{2}$ in the exterior potential $V$ as a function of the positions of the charges. This energy has a minimum, denoted by $E^{\mathrm{P}}(N, V)$ (with "P" denoting "particle"). A significant remark is that the charge configuration, for which the minimum is obtained, is confined within a radius independent of the total charge $N$ for fixed $V / N$. This "finite radius lemma", which also holds for the charge densities minimizing the functionals (1.3)-(1.6), is proved in an appendix. Using this and an electrostatics lemma of Lieb and Yau [26] we derive the bounds

$$
E^{\mathrm{C}}(N, V)-a N^{3 / 2} \geq E^{\mathrm{P}}(N, V) \geq E^{\mathrm{C}}(N, V)-b N^{3 / 2}
$$

where $a$ and $b$ depend only on $V / N$. These bounds are of independent interest apart from their role in the proof of the limit theorems where, in fact, only the latter inequality is needed. Upper and lower bounds to the quantum mechanical energy $E^{\mathrm{Q}}(N, B, V)$ in

terms of $E^{\mathrm{MTF}}(N, B, V)$ with controlled errors are derived in the final Section $\mathrm{V}$. The upper bound is a straightforward variational calculation using magnetic coherent states in the same way as in [21]. For the lower bound one treats the cases of large $B$ and small $B$ separately. The estimate for large $B$ is obtained by first noting that obviously $E^{\mathrm{Q}}(N, B, V) \geq E^{\mathrm{P}}(N, V)$, because the kinetic energy is nonnegative, and then using (1.18). For small $B$ two auxiliary results are required: A generalization of the magnetic Lieb-Thirring equality considered in [21], and an estimate of the correlation energy. Once these have been established the proof of Theorem 1.1 is completed by coherent state analysis. The limit theorem for the density follows easily from the limit theorem for the energy by perturbing $V$ with bounded functions.

\section{THE MTF THEORY: ITS DEFINITION AND PROPERTIES}

By employing the natural units defined in the introduction, the "kinetic energy" operator can be written 


$$
H_{\text {kin }}=\frac{1}{2}(i \nabla-\mathbf{A})^{2}+\gamma \mathbf{S} \cdot \mathbf{B}-\frac{1}{2}(1-|\gamma|) B
$$

with $\gamma=g_{*} m_{*} /(2 m)$. The spectrum of $H_{\text {kin }}$ is

$$
\varepsilon_{n, \sigma}=\left(n+\gamma \sigma+\frac{1}{2}|\gamma|\right) B
$$

with $n=0,1, \ldots, \sigma= \pm 1 / 2$. We write the energy levels (2.2) in strictly increasing order as $\varepsilon_{\nu}(B), \nu=0,1, \ldots$. The degeneracy of each level per unit area is $d_{\nu}(B)=B /(2 \pi)$, except if, by coincidence, $\gamma$ happens to be an integer; in that case $d_{\nu}(B)=B /(2 \pi)$ for $\nu=0, \ldots,|\gamma|-1$, while $d_{\nu}(B)$ is twice as large for the higher levels. It is worth recalling that if $V(x)=K|x|^{2}$, the spectrum of the one-body Hamiltonian $H_{1}$ in (1.2) is solvable. The spectrum of $H_{1}$ was determined by Fock [27] in 1928, two years before Landau's paper on the spectrum of $(i \nabla-\mathbf{A})^{2}$. For the Hamiltonian without spin, namely $\frac{1}{2}(i \nabla-\mathbf{A})^{2}+K|x|^{2}$, the spectrum is given by

$$
E=\frac{1}{2}\left(n_{1}-n_{2}\right) B+\frac{1}{2}\left(n_{1}+n_{2}+1\right)\left[4 K+B^{2}\right]^{1 / 2}
$$

with $n_{1}, n_{2}=0,1,2, \ldots$. It is remarkable that this simple spectrum gives a qualitatively good fit to some of the data [8].

For a gas of noninteracting fermions with the energy spectrum (2.2) the energy density $j_{B}$ as a function of the particle density $\rho$ is given by

$$
\begin{aligned}
& j_{B}(0)=0 \\
& j_{B}^{\prime}(\rho)=\varepsilon_{\nu}(B) \quad \text { if } \quad D_{\nu}(B)<\rho<D_{\nu+1}(B), \quad \nu=0,1 \ldots
\end{aligned}
$$

where $j_{B}^{\prime}=d j_{B} / d \rho$ and

$$
D_{\nu}(B)=\sum_{\nu^{\prime}=0}^{\nu} d_{\nu^{\prime}}(B)
$$


More explicitly,

$$
j_{B}(\rho)=\sum_{\nu=0}^{\nu_{\max }} \varepsilon_{\nu}(B) d_{\nu}(B)+\left(\rho-D_{\nu_{\max }}(B)\right) \varepsilon_{\nu_{\max }+1}(B)
$$

where $\nu_{\max }=\nu_{\max }(\rho, B)$ is defined by

$$
D_{\nu_{\max }}(B) \leq \rho<D_{\nu_{\max }+1}(B)
$$

Thus $j_{B}$ is a convex, piece-wise linear function with $j_{B}(\rho)=0$ for $0 \leq \rho \leq d_{1}(B)$. It has the scaling property

$$
j_{B}(\rho)=B^{2} j_{1}(\rho / B)
$$

As $B \rightarrow 0, j_{B}$ becomes a quadratic function of the density:

$$
\lim _{B \rightarrow 0} j_{B}(\rho)=j_{0}(\rho)=\frac{\pi}{2} \rho^{2} .
$$

Moreover,

$$
j_{B}(\rho) \leq j_{0}(\rho)
$$

for all $\rho$ and $B$ (see Fig. 1).

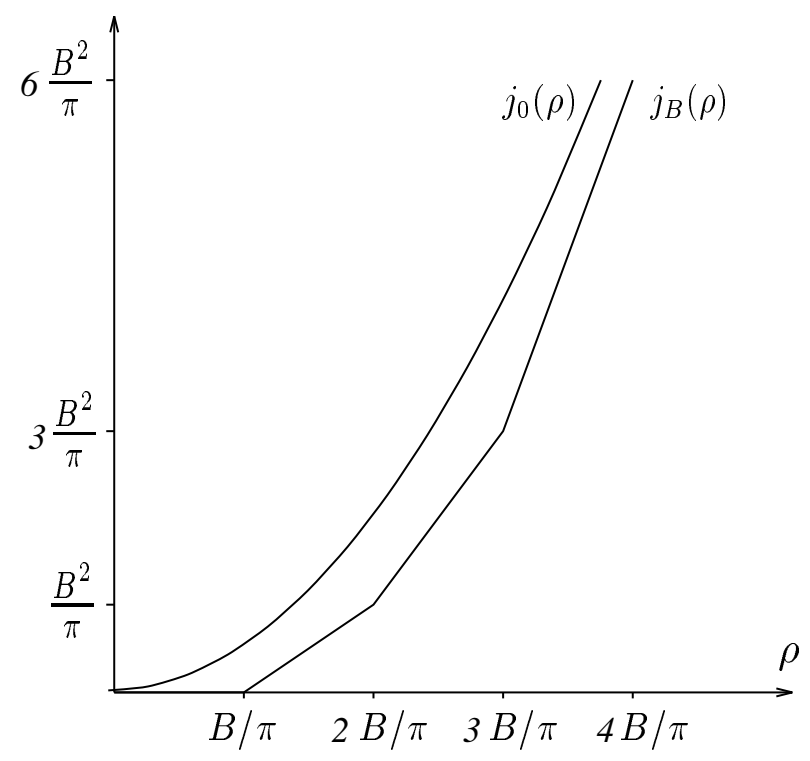

Fig. 1 The "kinetic" energy densities $j_{B}(\rho)$ and $j_{0}(\rho)$ in the special case where $\gamma=0$ 
Given an exterior potential $V$ the MTF functional is defined by (1.5). We assume that $V$ is continuous* and tends to $\infty$ as $|x| \rightarrow \infty$. In particular, $V$ is bounded below and, by adding a constant if necessary, we may assume that $V(x) \geq 0$ everywhere. Because of (2.6) the functional (1.5) is defined for all nonnegative functions $\rho$ such that $\int \rho V<\infty$, $\int \rho^{2}<\infty$ and $D(\rho, \rho)<\infty$. Since $V \geq 0$ the functional is nonnegative. If $N$ is some positive number we denote

$$
\mathcal{C}_{N}=\left\{\rho \mid \rho \geq 0, \int \rho V<\infty, \int \rho^{2}<\infty, D(\rho, \rho)<\infty, \int \rho=N\right\}
$$

and define the MTF energy by

$$
E^{\mathrm{MTF}}(N, B, V)=\inf _{\rho \in \mathcal{C}_{N}} \mathcal{E}[\rho ; B, V]
$$

Because of (2.4) the energy satisfies the scaling relation

$$
E^{\mathrm{MTF}}(N, B, V)=N^{2} E^{\mathrm{MTF}}(1, B / N, V / N) .
$$

In the limit $B \rightarrow 0$ the kinetic energy density $(2.3)$ converges to $j_{0}(\rho)=(\pi / 2) \rho^{2}$ and (1.5) converges to the energy functional (1.3) of two-dimensional TF theory at $B=0$. It is easy to see that also $\lim _{B \rightarrow 0} E^{\mathrm{MTF}}(N, B, V)=E^{\mathrm{TF}}(N, V)$, where $E^{\mathrm{TF}}$ is defined in the same way as $E^{\mathrm{MTF}}$ with (1.3) replacing (1.5). We can thus consider the TF theory as a special case of MTF theory. In the opposite limit, $B \rightarrow \infty$, the kinetic energy term vanishes altogether and one obtains a classical electrostatic model (1.6) that we shall study in Section III. Note also that since $j_{B} \leq j_{0}$ for all $B$ it follows that $E^{\mathrm{MTF}}(N, B, V) \leq$ $E^{\mathrm{TF}}(N, V)$ for all $B$. In particular $E^{\mathrm{MTF}}(1, \beta, v)$ is uniformly bounded in the parameter $\beta=B / N$ for fixed $v=V / N$.

\footnotetext{
* $V$ measurable and locally bounded would suffice
} 
For fixed $B$ and $V, E^{\mathrm{MTF}}(N, B, V)$ is a convex, continuously differentiable function of $N$ and, since $V \geq 0$, it is monotonically increasing. By the methods of [28], [29], [21] (see also [30]) it is straightforward to prove the existence and uniqueness of a minimizer for the variational problem (2.7).

2.1 THEOREM (Minimizer). There is a unique density $\rho_{N, B, V}^{\mathrm{MTF}} \in \mathcal{C}_{N}$ such that

$$
E^{\mathrm{MTF}}(N, B, V)=\mathcal{E}^{\mathrm{MTF}}\left(\rho_{N, B, V}^{\mathrm{MTF}}\right) .
$$

Note that the existence of a minimizing density with $\int \rho=N$ is guaranteed for all $N$ because $V(x) \rightarrow \infty$ as $|x| \rightarrow \infty$. This condition on $V$ also implies that $\rho_{N, B, V}$ vanishes outside a ball of finite radius, cf. Lemma A1 in the Appendix. The scaling relation for the minimizing density is

$$
\rho_{N, B, V}^{\mathrm{MTF}}(x)=N \rho_{1, B / N, V / N}^{\mathrm{MTF}}(x) .
$$

Theorem 2.1 includes the TF theory as a special case. In the same way as in Prop. 4.14 in [21] one shows that $\rho_{N, B, V}^{\mathrm{MTF}} \rightarrow \rho_{N, V}^{\mathrm{TF}}$ weakly in $L^{1}$ as $B \rightarrow 0$.

The shape of the electronic density in the case of a quadratic potential $V(x)=K|x|^{2}$ and $\gamma=0$ is shown in Fig. 2 for different values of $B$. The pictures were prepared by Kristinn Johnsen. At the highest value of $B(8 \mathrm{~T})$, the density is everywhere below $d_{0}(B)$ and given by the minimizer (3.15) of the classical functional (1.6). At $B=7 \mathrm{~T}$, all the electrons are still in the lowest Landau level, but that level is full around the middle of the dot where the density is anchored at $d_{0}(B)$. As the field gets weaker it becomes energetically favorable for electrons at the boundary of the dot, where the potential is high, to move into the next Landau level close to the minimum of the potential. A domeshaped region then arises above the plateau at $\rho=d_{0}(B)=D_{0}(B)$, but eventually the density hits the next plateau at $\rho=D_{1}(B)$. This gradual filling of Levels continues as the field strength goes down. At $B=2 \mathrm{~T}$ three Landau levels are full and electrons in the 
central dome are beginning to occupy the fourth level. Finally, at $B=0$, we have the usual Thomas-Fermi model, which may be regarded as a limiting case with infinitely many Landau levels occupied.
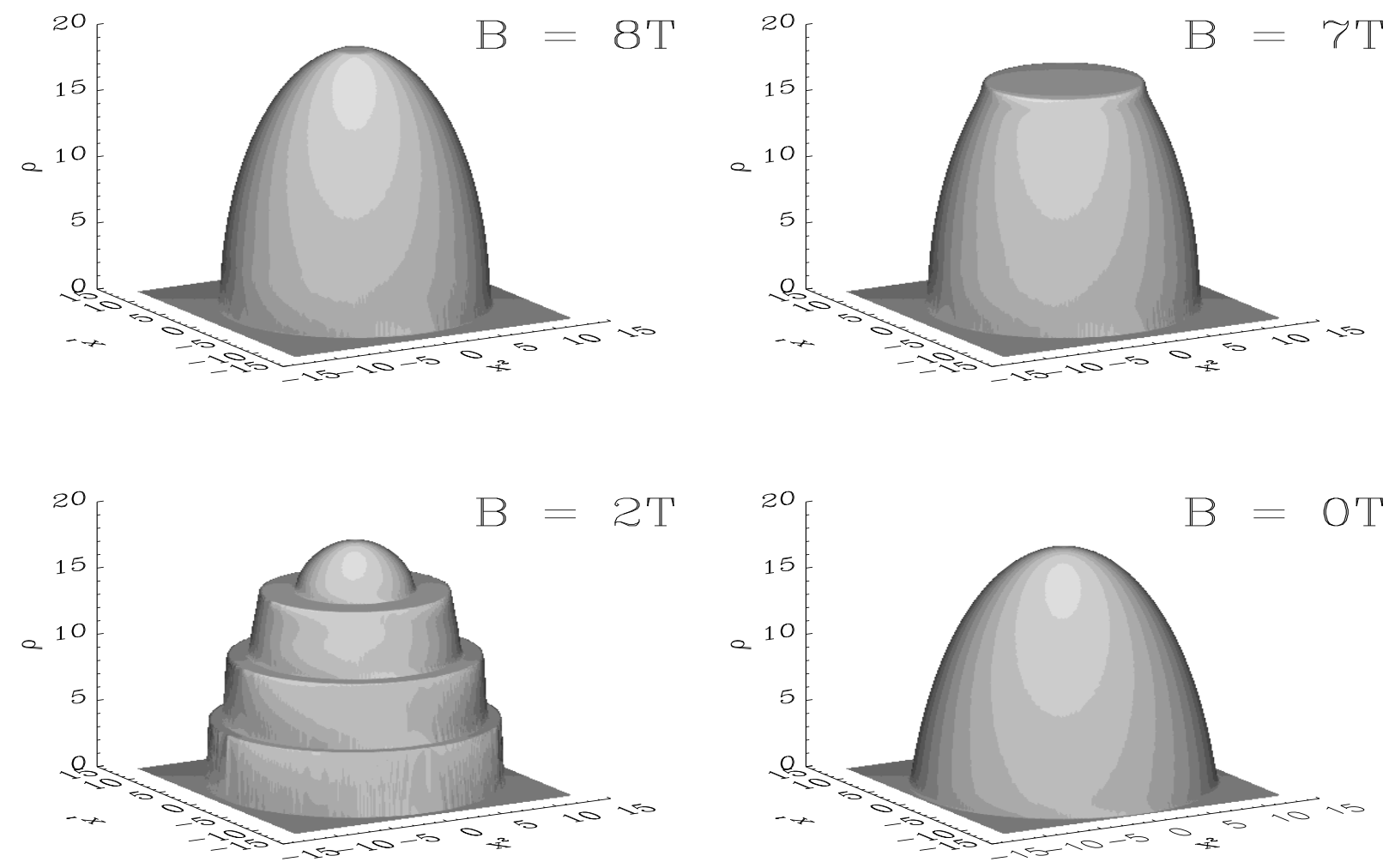

Fig. 2 Quantum dots at various magnetic field strengths. The potential is $V(x)=$ $(1 / 2) m_{*} \omega^{2}|x|^{2}$, with $m_{*}=0.67 m, \hbar \omega=3.37 \mathrm{meV}$, and $N=50$. The coordinate axes are displayed in units of $10^{-8} \mathrm{~m}$ and the density $\rho$ in the units $10^{-14} \mathrm{~m}^{-2}$.

In order to state the variational equation for the minimization problem it is convenient to define the derivative $j_{B}^{\prime}=d j_{B} / d \rho$ of the kinetic energy density everywhere, including points of discontinuity, as a set valued function (cf. [30]), namely

$$
j_{B}^{\prime}(\rho)= \begin{cases}\left\{\varepsilon_{\nu}(B)\right\} & \text { for } D_{\nu}(B)<\rho<D_{\nu+1}(B), \nu=0,1, \ldots \\ {\left[\varepsilon_{\nu}(B), \varepsilon_{\nu+1}(B)\right]} & \text { for } \rho=D_{\nu+1}(B), \nu=0,1, \ldots\end{cases}
$$


With this notation the Thomas-Fermi equation for the functional (1.5) may be written as follows

2.2 THEOREM (Thomas-Fermi equation). There is a nonnegative number $\mu=$ $\mu(N, B, V)$ such that the minimizer $\rho=\rho_{N, B, V}^{\mathrm{MTF}}$ satisfies

$$
\mu-V(x)-\rho *|x|^{-1}\left\{\begin{array}{ll}
\in j_{B}^{\prime}(\rho(x)) & \text { if } \rho(x)>0 \\
\leq 0 & \text { if } \rho(x)=0
\end{array} .\right.
$$

The quantity $\mu$ appearing in the TF equation is the physical chemical potential, i.e.,

$$
\mu=\partial E(N, B, V) / \partial N
$$

Since $E$ is convex as a function of $N, \mu$ is monotonically increasing with $N$ for fixed $B$ and $V$. It satisfies

$$
\mu(N, B, V)=N \mu(1, B / N, V / N)
$$

The derivation of the TF equation is analogous to that in [29]. It is also true that if $(\rho, \mu)$ is any solution pair for $(2.11)$, then $\rho$ is the minimizer of $\mathcal{E}$ for some $N$ and $\mu=\mu(N, B, V)$. The proof of this is a bit trickier than in the standard case [29], because $j_{B}$ is not continuously differentiable. It has been carried out by Lieb and Loss [30].

Finally we discuss the relationship between the MTF theory and the classical theory defined by the functional (1.6). We of course have that

$$
\mathcal{E}^{\mathrm{MTF}}[\rho ; B, V]=\int j_{B}(\rho(x)) \mathrm{d} x+\mathcal{E}^{\mathrm{C}}[\rho ; V]
$$

From the definition of $j_{B}$ one expects that the kinetic energy term above can be neglected for large $B$ and hence that $\lim _{B \rightarrow \infty} E^{\mathrm{MTF}}=E^{\mathrm{C}}$. The rigorous proof of this fact relies on a careful study of the classical problem. This analysis is far from trivial and is postponed to the next section. 
There is another case where the MTF energy can be related to the classical energy. Namely, for a homogeneous exterior potential, i.e.,

$$
V(\lambda x)=\lambda^{s} V(x)
$$

for all $\lambda>0$ with some $s>0$. We consider the potentials $k V(x)$ with $k>0$ and are interested in the dependence of the MTF energy and density on the coupling constant $k$.

Writing

$$
\rho(x)=k^{2 /(s+1)} \hat{\rho}\left(k^{1 /(s+1)} x\right)
$$

we have the scaling

$$
\begin{aligned}
\mathcal{E}^{\mathrm{MTF}}[\rho ; B, k V] & =k^{2 /(s+1)} \int j_{b}(\hat{\rho})+\mathcal{E}^{\mathrm{C}}[\rho ; k V] \\
& =k^{1 /(s+1)}\left[k^{1 /(s+1)} \int j_{b}(\hat{\rho})+\mathcal{E}^{\mathrm{C}}[\hat{\rho} ; V]\right]
\end{aligned}
$$

where

$$
b=B k^{-2 /(s+1)}
$$

Changing $k$ is thus equivalent to changing the kinetic energy by a multiplicative factor and rescaling the magnetic field, keeping the potential fixed. We shall show in the next section that for $k$ small $E^{\mathrm{C}}$ is a good approximation to $E^{\mathrm{MTF}}$.

\section{THE CLASSICAL CONTINUOUS MODEL: A LIMIT OF MTF THEORY}

For densities $\rho$ small enough $\left(\rho(x) \leq d_{1}(B)\right.$ for all $\left.x\right)$ the kinetic energy $j_{B}(\rho)$ vanishes. It is therefore natural to consider the resulting classical energy functional defined by (1.6), i.e.,

$$
\mathcal{E}^{\mathrm{C}}[\rho ; V]=\int \rho(x) V(x) \mathrm{d} x+\frac{1}{2} \iint \rho(x)|x-y|^{-1} \rho(y) \mathrm{d} x \mathrm{~d} y
$$


The corresponding classical energy is

$$
E^{\mathrm{C}}(N, V)=\inf \left\{\mathcal{E}^{\mathrm{C}}[\rho ; V]: \rho \geq 0, \int \rho=N\right\}
$$

In this section we analyze this functional and prove that it is, indeed, the large $B$ limit of MTF theory.

As before we assume that the confining potential $V$ is positive and that $V(x) \rightarrow \infty$ as $|x| \rightarrow \infty$. Moreover, we shall here assume that $V$ is continuous (in fact, we shall make an even more stringent regularity assumption in Theorem 3.2 below).

We begin by showing the existence of a minimizer for (3.2). For general continuous $V$ (without further assumptions) we must take into account the possibility that the minimizing $\rho$ may be a measure. In (3.2) we therefore minimize over all positive measures $\rho$ with $\int \rho=N$. It follows from the finite radius lemma given in the appendix that

$$
E^{\mathrm{C}}(N, V)=\inf \left\{\mathcal{E}^{\mathrm{C}}[\rho, V]: \text { support } \rho \subset\left\{x:|x| \leq R_{v}\right\}, \rho \geq 0, \quad \rho=N\right\}
$$

Here $R_{v}$ depends only on $v=V / N$. Later on we shall show that the minimizer is, indeed, a function, and hence that (3.2) does give us the large $B$ limit of MTF theory for suitable $V$.

3.1. PROPOSITION (Existence and uniqueness of a minimizing measure). Let $V$ be continuous. Then there is a unique positive measure $\rho_{N, V}^{\mathrm{C}}$ with $\int \rho_{N, V}^{\mathrm{C}}=N$ such that $E^{\mathrm{C}}(N, V)=\mathcal{E}^{\mathrm{C}}\left[\rho_{N, V}^{\mathrm{C}} ; V\right]$

Proof: [Note: we write measures as $\rho(x) \mathrm{d} x$, even if they are not absolutely continuous with respect to Lebesgue measure.] By (3.3) we can choose a sequence of positive measures, $\rho_{1}, \rho_{2}, \ldots$, supported in $\left\{x:|x| \leq R_{v}\right\}$ with $\int \rho_{n}=N$, such that $\lim _{n \rightarrow \infty} \mathcal{E}^{\mathrm{C}}\left[\rho_{n} ; V\right]=$ $E^{\mathrm{C}}(N, V)$. The bounded measures are the dual of the continuous functions, and so, by the Banach-Alaoglu Theorem, we may assume (by possibly passing to a subsequence) that $\rho_{n}$ 
converges weakly to a positive measure $\rho$ still supported in $\left\{x:|x| \leq R_{v}\right\}$. In particular it follows that $\int \rho=N$ and $\int \rho_{n} V \rightarrow \int \rho V$. Moreover, the product measure $\rho_{n} \times \rho_{n} \rightarrow \rho \times \rho$ weakly. Hence

$$
\begin{aligned}
\iint \rho(x) \rho(y)|x-y|^{-1} \mathrm{~d} x \mathrm{~d} y & =\lim _{\delta \rightarrow 0} \iint \rho(x) \rho(y)(|x-y|+\delta)^{-1} \mathrm{~d} x \mathrm{~d} y \\
& =\lim _{\delta \rightarrow 0} \lim _{n \rightarrow \infty} \iint \rho_{n}(x) \rho_{n}(y)\left(|x-y|^{-1}+\delta\right)^{-1} \mathrm{~d} x \mathrm{~d} y \\
& \leq \liminf _{n \rightarrow \infty} \iint \rho_{n}(x) \rho_{n}(y)|x-y|^{-1} \mathrm{~d} x \mathrm{~d} y .
\end{aligned}
$$

The first equality follows by the Lebesgue's monotone convergence Theorem. The last inequality is an immediate consequence of the pointwise bound $(|x-y|+\delta)^{-1} \leq|x-y|^{-1}$.

We conclude from (3.4) that

$$
E^{\mathrm{C}}(N, V) \leq \mathcal{E}^{\mathrm{C}}[\rho ; V] \leq \liminf _{n \rightarrow \infty} \mathcal{E}^{\mathrm{C}}\left[\rho_{n} ; V\right]=E^{\mathrm{C}}(N, V)
$$

and hence that $\rho$ is a minimizer.

The uniqueness of $\rho$ follows from strict convexity of $D(\rho, \rho)$.

Q.E.D.

The next theorem gives conditions, which are perfectly adequate for the physical applications, under which the minimizer is a function and not just a measure. Moreover, that function has certain nice integrability properties.

3.2 THEOREM (The minimizer is a function). Assume that the potential $V$ is in the class $C_{\mathrm{loc}}^{1, \alpha}$ for some $0<\alpha \leq 1$. (I.e., $V$ is once continuously differentiable and for each $R>0$ its derivative satisfies

$$
|\nabla V(x)-\nabla V(y)| \leq c_{R}|x-y|^{\alpha}
$$

inside the ball of radius $R$ centered at the origin for some constant $c_{R}>0$.) Then the minimizing measure $\rho_{N, V}^{\mathrm{C}}$ of Prop. 3.1 is a function. It has the properties (with $\widehat{\rho}$ being 
the Fourier transform of $\rho$ )

$$
\begin{aligned}
& \int\left|\widehat{\rho}_{N, V}^{\mathrm{C}}(p)\right|^{2}|p|^{r} \mathrm{~d} p<C_{1}, \quad-1 \leq r<\alpha \\
& \rho_{N, V}^{\mathrm{C}} *|x|^{-1} \text { is continuous } \\
& \quad \int \rho_{N, V}^{\mathrm{C}}(x)^{q} \mathrm{~d} x<C_{2}, \quad 1 \leq q<\frac{4}{2-\alpha}
\end{aligned}
$$

where $C_{1}$ and $C_{2}$ are constants (implicitly computed below) that depend only on the constants $c_{R}, q, r, \alpha$ and on $N$.

Proof: We write $\rho_{N, V}^{\mathrm{C}}=\rho$. We know that $\int \rho=N$ and that $\rho$ has compact support. From the former fact we conclude that $\widehat{\rho}$ is well defined, continuous and bounded.

Let $g_{a}$ be the function with Fourier transform

$$
\widehat{g}_{a}(p)=\int g_{a}(x) e^{i p x} \mathrm{~d} x= \begin{cases}1, & |p| \leq a \\ 0, & |p| \geq a\end{cases}
$$

Then $g_{a}$ is continuous, $\int g_{a}=1$ and $\int y g_{a}(y) \mathrm{d} y=0$.

Let $\rho_{a}$ be the convolution $\rho * g_{a}$, so that $\int \rho_{a}=N$.

Since $\rho$ is a minimizer, $\mathcal{E}^{\mathrm{C}}[\rho ; V] \leq \mathcal{E}^{\mathrm{C}}\left[\rho_{a} ; V\right]$. Explicitly this inequality is

$$
\int\left(\rho_{a}-\rho\right) V+D\left(\rho_{a}, \rho_{a}\right)-D(\rho, \rho) \geq 0
$$

Since $\int V \rho_{a}=\int\left(V * g_{a}\right) \rho$ we can rewrite the first term as

$$
\iint[V(x-y)-V(x)] \rho(x) g_{a}(y) \mathrm{d} x \mathrm{~d} y .
$$

By integrating $\nabla V$ along the line from $x-y$ to $x$, and using (3.6), we have

$$
|V(x-y)-V(x)+y \cdot \nabla V(x)| \leq c|y|^{\alpha+1}
$$

(Note that by the finite radius Lemma A1 all integrals are restricted to a finite ball.) Using that $\int y g_{a}(y)=0$ we can estimate the first term in (3.11) as follows:

$$
\int\left(\rho_{a}-\rho\right) V \leq C \int|y|^{\alpha+1}\left|g_{a}(y)\right| \mathrm{d} y=(\text { const. }) a^{-\alpha-1}
$$


The last two terms in (3.11) are

$$
\text { (const.) } \int|\widehat{\rho}(p)|^{2}|p|^{-1}\left[\left|\widehat{g}_{a}(p)\right|^{2}-1\right] \mathrm{d} p=(\text { const. }) \int_{|p| \geq a}|\widehat{\rho}(p)|^{2}|p|^{-1} \mathrm{~d} p
$$

(Recall that $\mid \widehat{\left.x\right|^{-1}}=$ (const.) $|p|^{-1}$ in two-dimensions.) The inequality (3.11) thus implies

$$
\int_{|p| \geq a}|\widehat{\rho}(p)|^{2}|p|^{-1} \mathrm{~d} p \leq(\text { const. }) a^{-\alpha-1} .
$$

Using (3.12) and $\widehat{\rho}(p) \leq \int \rho=N$ we can now prove (3.7) as follows.

$$
\begin{aligned}
\int|\widehat{\rho}(p)|^{2}|p|^{r} \mathrm{~d} p & =\int_{|p| \leq 1}|\widehat{\rho}(p)|^{2}|p|^{r} \mathrm{~d} p+\sum_{n=0}^{\infty} \int_{2^{n} \leq|p| \leq 2^{n+1}}|\widehat{\rho}(p)|^{2}|p|^{r} \mathrm{~d} p \\
& \leq N^{2} \int_{|p| \leq 1}|p|^{r} \mathrm{~d} p+\text { (const.) } \sum_{n=0}^{\infty} 2^{(r+1)(n+1)} \int_{2^{n} \leq|p| \leq 2^{n+1}}|\widehat{\rho}(p)|^{2}|p|^{-1} \mathrm{~d} p \\
& \leq \text { (const.) } N^{2}+\text { (const.) } \sum_{n=0}^{\infty} 2^{(r+1)(n+1)-n(\alpha+1)}<\infty,
\end{aligned}
$$

if $r<\alpha$.

To prove that $\rho *|x|^{-1}$ is continuous is now easy. We simply prove that its Fourier transform is integrable. The Fourier transform of $\rho *|x|^{-1}$ is (const.) $\widehat{\rho}(p)|p|^{-1}$ and

$$
\int|\widehat{\rho}(p)||p|^{-1} \mathrm{~d} p \leq \int_{|p| \leq 1}|\widehat{\rho}(p)||p|^{-1} \mathrm{~d} p+\left(\int_{|p| \geq 1}|\widehat{\rho}(p)|^{2}|p|^{r} \mathrm{~d} p\right)^{1 / 2}\left(\int_{|p| \geq 1}|p|^{-r-2} \mathrm{~d} p\right)^{1 / 2}<\infty .
$$

Finally, we prove (3.9). For $1 \leq q \leq 2$ there is no problem because we know that $\int \rho^{2}=\int \widehat{\rho^{2}}$. Hence $\rho$ is a square integrable function and, since $\int \rho=N$, we conclude by Hölder's inequality that (3.9) holds for $1 \leq q \leq 2$. For $q>2$ we will prove that

$$
\int|\widehat{\rho}|^{t}<\infty \text { with } \frac{4}{\alpha+2}<t=\frac{q}{q-1}<2
$$

This will prove $(3.9)$ by the Hausdorff-Young inequality, which states that $\left(\int|\widehat{\rho}|^{t}\right)^{1 / t} \geq$ $\left(\int \rho^{q}\right)^{1 / q}$ when $1 \leq t \leq 2$. 
We write $|\widehat{\rho}(p)|^{t}=\left\{|\widehat{\rho}(p)|^{t}(1+|p|)^{m}\right\}\left\{(1+|p|)^{-m}\right\}$ and then use Hölder's inequality with $a^{-1}+b^{-1}=1$ to conclude that

$$
\int|\widehat{\rho}|^{t} \leq\left[\int|\widehat{\rho}(p)|^{t a}(1+|p|)^{m a} \mathrm{~d} p\right]^{1 / a}\left[\int(1+|p|)^{-m b} \mathrm{~d} p\right]^{1 / b}
$$

Thus $\int|\widehat{\rho}|^{t}<\infty$ if we can satisfy $t a=2, m a<\alpha$ and $m b>2$, in addition to $a^{-1}+b^{-1}=1$. This requires $\alpha / a>m>2 / b$, or $1 \leq a<1+\frac{1}{2} \alpha$. Thus, we require $t=2 / a>4 /(\alpha+2)$ which, since $q=t /(t-1)$, means $q<4 /(2-\alpha)$.

Q.E.D.

Corresponding to the minimization (3.2) there is a variational "equation" satisfied by the minimizer $\rho$. In the general case, in which $\rho$ might be a measure, the variational equation exists but is slightly complicated to state.

In physically interesting cases $V$ is certainly in $C^{1, \alpha}$, in which case Theorem 3.2 tells us that $\rho$ is a function and that $\rho *|x|^{-1}$ is continuous. Hence the total potential

$$
V^{\mathrm{C}}=V+\rho *|x|^{-1}
$$

is continuous. It is then easy to derive by standard arguments, as in Sect. 1, that $\rho$ is the unique nonnegative solution to the variational equation

$$
\begin{aligned}
& V(x)+\rho *|x|^{-1}=\mu \quad \text { if } \quad \rho(x) \neq 0 \\
& V(x)+\rho *|x|^{-1} \geq \mu \quad \text { if } \quad \rho(x)=0,
\end{aligned}
$$

for a unique $\mu>0$. As usual the chemical potential $\mu$ is a monotone function of the particle number $N=\int \rho$. In the special case of a parabolic confining potential the solution to (3.14) can be given in closed form.

3.3 PROPOSITION (Minimizer for the parabolic exterior potential). If $V(x)=K|x|^{2}$ then the minimizer of $\mathcal{E}_{N, V}^{\mathrm{C}}$ is

$$
\rho_{N, V}^{\mathrm{C}}(x)=\left\{\begin{array}{ll}
\frac{3}{2 \pi} N \lambda \sqrt{1-\lambda|x|^{2}}, & \text { if }|x| \leq \lambda^{-1} \\
0, & \text { if }|x|>\lambda^{-1}
\end{array},\right.
$$


where $\lambda=(8 K / 3 \pi N)^{2 / 3}$. In fact, $\rho_{N, V}^{\mathrm{C}}$ is the solution to (3.14) with $\mu=(3 \pi / 4) N \lambda^{1 / 2}$.

Proof: This solution (3.15) was certainly known before; see e.g., [10]. We give the proof here for the convenience of the reader. We only have to show that $\rho=\rho_{N, V}^{\mathrm{C}}$ is the solution to (3.14). It is enough to consider the case $\lambda=1$ and $N=1$. Then $V(x)=\frac{3 \pi}{8}|x|^{2}$ and $\mu=\frac{3 \pi}{4}$. We may compute $\rho *|x|^{-1}=\frac{3}{2 \pi} \int \sqrt{1-|x-y|^{2}}|y|^{-1} \mathrm{~d} y$ by writing $y$ in polar coordinates $(|y|, \theta)$ and performing the $|y|$ integration first.

$$
\begin{aligned}
\rho *|x|^{-1} & =\frac{3}{2 \pi} \iint\left[(1-|y|-|x| \cos \theta)^{2}-|x|^{2} \sin ^{2} \theta\right]^{1 / 2} \mathrm{~d} \theta \mathrm{d}|y| \\
& =\frac{3}{2 \pi} \int\left(1-|x|^{2} \sin ^{2} \theta\right)^{1 / 2} \int\left(1-\frac{(|y|-|x| \cos \theta)^{2}}{1-|x|^{2} \sin ^{2} \theta}\right)^{1 / 2} \mathrm{~d}|y| \mathrm{d} \theta,
\end{aligned}
$$

the integrations are over the intervals in $\theta$ and $|y|$ for which the integrands are real. Introducing the variable $t=(|y|-|x| \cos \theta)\left(1-|x|^{2} \sin ^{2} \theta\right)^{-1 / 2}$ we obtain

$$
\begin{aligned}
\rho *|x|^{-1} & =\frac{3}{2 \pi} \int_{-\theta_{m}(x)}^{\theta_{m}(x)}\left(1-|x|^{2} \sin ^{2} \theta\right) \mathrm{d} \theta \int_{-1}^{1}\left(1-t^{2}\right)^{1 / 2} \mathrm{~d} t \\
& =\frac{3}{4} \int_{-\theta_{m}(x)}^{\theta_{m}(x)}\left(1-|x|^{2} \sin ^{2} \theta\right) d \theta
\end{aligned}
$$

where

$$
\theta_{m}(x)= \begin{cases}\pi / 2, & \text { if }|x| \leq 1 \\ \sin ^{-1} \frac{1}{|x|}, & \text { if }|x|>1\end{cases}
$$

Thus

$$
\rho *|x|^{-1}=\frac{3 \pi}{4}-\frac{3 \pi}{8}|x|^{2} \quad \text { if }|x| \leq 1
$$

and

$$
\rho *|x|^{-1} \geq \frac{3 \pi}{4}-\frac{3 \pi}{8}|x|^{2} \quad \text { if }|x|>1
$$

(The last inequality comes from the fact that the integral from $\theta_{m}$ to $\pi / 2$ in $(3.16)$ is negative.)

Q.E.D. 
The energy function $E^{\mathrm{C}}(N, V)$ has the simple scaling:

$$
E^{\mathrm{C}}(N, V)=N^{2} E^{\mathrm{C}}\left(1, \frac{V}{N}\right) .
$$

The minimizing density $\rho_{N, V}^{\mathrm{C}}$ for $(3.2)$ scales as

$$
\rho_{N, V}^{\mathrm{C}}(x)=N \rho_{1, v}^{\mathrm{C}}(x)
$$

where $v=V / N$.

We shall now make precise in what sense the classical energy $E^{\mathrm{C}}$ is a limit of the MTF energy. In fact, in two different limits (the large $B$ limit and the low coupling limit) the MTF energy will converge to the classical energy. We first treat the large $B$ limit.

3.4 THEOREM (Large $B$ limit of MTF). If the exterior potential $V$ is in the class $C_{\text {loc }}^{1, \alpha}$ we have, as $B \rightarrow \infty$,

$$
E^{\mathrm{MTF}}(N, B, V) \rightarrow E^{\mathrm{C}}(N, V)
$$

and

$$
\rho_{N, B, V}^{\mathrm{MTF}}(x) \rightarrow \rho_{N, V}^{\mathrm{C}}
$$

in the weak $L^{1}$ sense.

Proof: If we use $\rho_{N, B, V}^{\mathrm{MTF}}$ as a trial density in $\mathcal{E}^{\mathrm{C}}$ and recall that $j_{B} \geq 0$ we immediately obtain $E^{\mathrm{C}}(N, V) \leq E^{\mathrm{MTF}}(N, B, V)$.

For the bound in the opposite direction we use $\rho_{N, V}^{\mathrm{C}}$ as a trial density for $\mathcal{E}^{\mathrm{MTF}}$. In order to do this it is, however, important that we know (from Theorem 3.2) that $\rho_{N, V}^{\mathrm{C}}$ is a function. Hence $j_{B}\left(\rho_{N, V}^{\mathrm{C}}\right)$ is well defined. Moreover, from the definition of $j_{B}, j_{B}\left(\rho_{N, V}^{\mathrm{C}}\right) \rightarrow$ 0 almost everywhere as $B \rightarrow \infty$ and $j_{B}\left(\rho_{N, V}^{\mathrm{C}}\right) \leq j_{0}\left(\rho_{N, V}^{\mathrm{C}}\right)$. Since $j_{0}(\rho)=(\pi / 2)(\rho)^{2}$ we know from (3.9) that $j_{0}\left(\rho_{N, V}^{\mathrm{C}}\right)$ is integrable. The limit in (3.18) is therefore an immediate consequence of Lebesgue's dominated convergence theorem. 
The convergence of the densities in (3.19) follows in a standard way by replacing $V$ by $V+\varepsilon f$ with $f$ a bounded (measurable) function and differentiating w.r.t. $\varepsilon$, see e.g., $[29]$.

Q.E.D.

We point out that if $\rho_{N, V}^{\mathrm{C}}$ is a bounded function, as it is, e.g., for $V=K|x|^{2}$, then

$$
E^{\mathrm{MTF}}(N, B, V)=E^{\mathrm{C}}(N, V)
$$

for $B$ large enough, because in that case $j_{B}\left(\rho_{N, V}^{\mathrm{C}}\right)$ vanishes for $B$ large.

Finally, we now discuss the weak coupling limit in the case of homogeneous exterior potentials. Suppose $V$ is a homogeneous function of $x, V(\lambda x)=\lambda^{s} V(x), s>0$. If we consider the exterior potentials $k V(x)$ with $k>0$ the classical energy and density obey the scalings

$$
E^{\mathrm{C}}(N, k V)=k^{1 /(s+1)} E^{\mathrm{C}}(N, V)
$$

and

$$
\rho_{N, k V}^{\mathrm{C}}(x)=k^{2 /(s+1)} \rho_{N, V}^{\mathrm{C}}\left(k^{1 /(s+1)} x\right) .
$$

If $k$ is small we see from (3.22) that the minimizing density for the MTF functional will spread out and its kinetic energy will be negligible compared with the classical terms. We prove this rigorously now.

3.5 THEOREM (Weak coupling limit of MTF with homogeneous potentials). Let V be $C_{\mathrm{loc}}^{1, \alpha}$ and homogeneous of degree $s$. If $k \rightarrow 0$ then

$$
\frac{E^{\mathrm{MTF}}(N, B, k V)}{E^{\mathrm{C}}(N, k V)} \rightarrow 1
$$

and

$$
k^{-2 /(s+1)} \rho_{N, B, k V}^{\mathrm{MTF}}\left(k^{-1 /(s+1)} x\right) \rightarrow \rho_{N, V}^{\mathrm{C}}(x),
$$

in weak $L^{1}$ sense. Both limits are uniform in $B$. 
Proof: As above we may use $\rho_{N, B, V}^{\mathrm{MTF}}$ as a trial density in $\mathcal{E}^{\mathrm{C}}$ to conclude that $E^{\mathrm{C}}(N, V) \leq$ $E^{\mathrm{MTF}}(N, B, V)$.

To prove the bound in the opposite direction we again use $\rho_{N, V}^{\mathrm{C}}$ as a trial density for $\mathcal{E}^{\mathrm{MTF}}$. We then obtain from $(2.15)$ and the scaling $(3.22)$ that

$$
E^{\mathrm{MTF}}(N, B, k V) \leq k^{2 /(s+1)} \int j_{0}\left(\rho_{N, V}^{\mathrm{C}}\right)+E^{\mathrm{C}}(N, k V)
$$

where we used that $j_{b} \leq j_{0}$. If we compare this with the scaling in (3.21) we see that $E^{\mathrm{MTF}} / E^{\mathrm{C}} \rightarrow 1$ as $k \rightarrow 0$ since $j_{0}\left(\rho_{N, V}^{\mathrm{C}}\right)=(\pi / 2)\left(\rho_{N, V}^{\mathrm{C}}\right)^{2}$ is integrable.

The convergence of the densities follows again by replacing $V$ by $V+\varepsilon f$ and differentiating w.r.t. $\varepsilon$.

Q.E.D.

In the same way as for the large $B$ limit (3.23) becomes an identity for small $k$ if $\rho_{N, V}^{\mathrm{C}}$ is a bounded function.

We may of course also introduce the scaling $V=N v$ when $v$ is homogeneous of degree $s$. Then $k V=K v$, where $K=k N$, and the limit in (3.23) is uniform in $N$. The limit in (3.24) is uniform if we formulate it as

$$
N^{-1} k^{-2 /(s+1)} \rho_{N, B, k V}^{\mathrm{MTF}}\left(k^{-1 /(s+1)} x\right) \rightarrow \rho_{1, v}^{\mathrm{C}}(x)
$$

We remark that if a potential $W$ is asymptotically homogeneous in the sense that there is a homogeneous potential $V$ with $\lim _{|x| \rightarrow \infty} W(x) / V(x)=1$, then

$$
\lim _{k \rightarrow 0} E(N, B, k W) / k^{1 /(1+s)}=E^{\mathrm{C}}(N, V)
$$

uniformly in $B$, where $s$ is the degree of homogeneity of $V$.

\section{THE CLASSICAL POINT CHARGE MODEL: A LIMIT OF QUANTUM MECHANICS}


Another model that sheds some light on the physics of our problem — and that will also be important for bounding the difference between the TF theory and the original quantum theory in Sect. V - is the classical particle model. In this model the kinetic energy is simply omitted altogether, but the point-like nature of the electrons is retained.

4.1. DEFINITION (Classical particle energy). With $V(x)$ being the confining potential the classical particle energy for $N$ points in $\mathbf{R}^{2}$ is defined by

$$
\mathcal{E}^{\mathrm{P}}\left(x_{1}, \ldots, x_{N} ; V\right)=\sum_{i=1}^{N} V\left(x_{i}\right)+\sum_{1 \leq i<j \leq N}\left|x_{i}-x_{j}\right|^{-1} .
$$

The minimum classical particle energy for $N$ point particles in $\mathbf{R}^{2}$ is

$$
E^{\mathrm{P}}(N, V)=\inf \left\{\mathcal{E}^{\mathrm{P}}\left(x_{1}, \ldots, x_{N} ; V\right): x_{i} \in \mathbf{R}^{2}\right\}
$$

We shall estimate the particle energy $E^{\mathrm{P}}(N, V)$ in terms of the classical continuum energy $E^{\mathrm{C}}(N, V)$. We first show that $E^{\mathrm{C}}(N, V)$ gives an exact upper bound on $E^{\mathrm{P}}(N, V)$.

4.2. LEMMA (Upper bound for $E^{\mathrm{P}}$ ). For all $N$ we have

$$
E^{\mathrm{P}}(N, V) \leq E^{\mathrm{C}}(N, V)-N^{3 / 2} /\left(8 R_{v}\right),
$$

where $R_{v}$ is the maximal radius given in Lemma $A 1$.

Proof: First, let us give a very simple argument that yields an error term proportional to $N$ instead of $N^{3 / 2}$. The energy $E^{\mathrm{P}}(N, V)$ is bounded above by

$$
\int \mathcal{E}^{\mathrm{P}}\left(x_{1}, \ldots, x_{N} ; V\right) \Phi\left(x_{1}, \ldots, x_{N}\right) \mathrm{d} x_{1} \ldots \mathrm{d} x_{N}
$$

for any nonnegative function $\Phi$ with $\int \Phi=1$. We take $\Phi\left(x_{1}, \ldots, x_{N}\right)=\prod_{i=1}^{N} \rho_{[1]}\left(x_{i}\right)$, where for simplicity we have introduced the notation $\rho_{[1]}$ for the minimizer $\rho_{1, V / N}^{\mathrm{C}}$ for $\mathcal{E}^{\mathrm{C}}[\rho ; V / N]$ with $\int \rho_{1, V / N}^{\mathrm{C}}=1$. Note that $\rho_{[1]}$ depends only on $v=V / N$. We obtain

$$
\begin{aligned}
E^{\mathrm{P}}(N, V) & \leq \int \mathcal{E}^{\mathrm{P}}\left(x_{1}, \ldots, x_{N} ; V\right) \prod_{i=1}^{N} \rho_{[1]}\left(x_{i}\right) d x_{1} \ldots d x_{N} \\
& =N \int V(x) \rho_{[1]}(x) \mathrm{d} x+\frac{N(N-1)}{2} \iint \rho_{[1]}(x)|x-y|^{-1} \rho_{[1]}(y) \mathrm{d} x \mathrm{~d} y
\end{aligned}
$$


Recalling that the minimizer of $\mathcal{E}^{\mathrm{C}}$ is $\rho_{N, V}^{\mathrm{C}}(x)=N \rho_{[1]}(x)$, we get an error term $-a N$, with $a=\frac{1}{2} \iint \rho_{[1]}(x) \rho_{[1]}(y)|x-y|^{-1} \mathrm{~d} x \mathrm{~d} y$.

Now we turn to a proof of (4.3) which, obviously, has to be more complicated than the previous discussion. By Lemma A1 there is a fixed square, $Q$, centered at the origin, whose width, $W$, equals $2 R_{v}$, such that the minimizer $\rho=\rho_{N, V}^{\mathrm{C}}$ for $\mathcal{E}^{\mathrm{C}}$ is supported in $Q$. For simplicity we suppose that $\sqrt{N}$ is an integer; if this is not so the following proof can be modified in an obvious way.

First, cut $Q$ into $\sqrt{N}$ vertical, disjoint strips, $S_{1}, S_{2}, \ldots, S_{\sqrt{N}}$ such that $\int_{S_{j}} \rho=\sqrt{N}$ for all $j$. Let $t_{j}$ denote the width of $S_{j}$, so that $\sum_{j=1}^{\sqrt{N}} t_{j}=W$. Next, make $\sqrt{N}-1$ horizontal cuts in each $S_{j}$ so that the resulting rectangles $R_{j k}$ for $k=1, \ldots, \sqrt{N}$ satisfy $\int_{R_{j k}} \rho=1$. Denote the height of these rectangles by $h_{j k}$, so that $\sum_{k=1}^{\sqrt{N}} h_{j k}=W$ for each $j$. Having done this we note, by convexity, that for each $j$

$$
N^{-1 / 2} \sum_{k=1}^{\sqrt{N}}\left(t_{j}+h_{j k}\right)^{-1} \geq\left[N^{-1 / 2} \sum_{k=1}^{\sqrt{N}}\left(t_{j}+h_{j k}\right)\right]^{-1}=\left[t_{j}+N^{-1 / 2} W\right]^{-1}
$$

Again, using the same convexity argument for the $j$-summation, we have that

$$
\sum_{j=1}^{\sqrt{N}} \sum_{k=1}^{\sqrt{N}}\left(t_{j}+h_{j k}\right)^{-1} \geq \frac{N^{3 / 2}}{2 W}
$$

Let $\rho_{j k}$ be the minimizing density $\rho$ restricted to the rectangle $R_{j k}$, i.e. $\rho_{j k}(x)=$ 1 if $x \in R_{j k}$ and $=0$ otherwise. Thus, $\int \rho_{j k}=1$. We denote these $N$ function by $\rho^{i}, i=1, \ldots, N$. Define $\Phi\left(x_{1}, \ldots, x_{N}\right):=\prod_{i=1}^{N} \rho^{i}\left(x_{i}\right)$ and, as in the previous proof, a simple computation yields

$$
E^{\mathrm{P}}(N, V) \leq \int \mathcal{E}^{\mathrm{P}} \Phi=\mathcal{E}^{\mathrm{C}}[\rho ; V]-\sum_{i=1}^{N} D\left(\rho^{i}, \rho^{i}\right)
$$

with $D(f, g)=\frac{1}{2} \iint f(x) g(y)|x-y|^{-1} \mathrm{~d} x \mathrm{~d} y$. 
To complete our proof we note that as long as $x$ and $y$ are in $R_{j k}$ we have that $|x-y|^{-1} \geq\left(t_{j}+h_{j k}\right)^{-1}$. Thus, $\sum_{j, k} D\left(\rho_{j k}, \rho_{j k}\right) \geq N^{3 / 2} / 4 W$ by (4.4) and the fact that $\int \rho_{j k}=1$.

Q.E.D.

4.3. LEMMA (Lower bound for $\mathrm{E}^{\mathrm{P}}$ ). Assume that $V$ is a potential in $C_{\mathrm{loc}}^{1, \alpha}$. Then for all $N$ we have

$$
E^{\mathrm{P}}(N, V) \geq E^{\mathrm{C}}(N, V)-b N^{3 / 2}
$$

with

$$
b=\frac{4}{3} \sqrt{\frac{2}{3}} \int\left(\rho_{1, v}^{\mathrm{C}}(x)\right)^{3 / 2} \mathrm{~d} x+\left(\frac{2 \pi}{2-p}\right)^{1 / p}\left(2 R_{v}\right)^{-1+(2 / p)}\left(\int\left(\rho_{1, v}^{\mathrm{C}}\right)^{q}\right)^{1 / q}
$$

and where $q$ is any number satisfying $2<q<4 /(2-\alpha), R_{v}$ is the maximal radius given in Lemma A1 and $p=q /(q-1)<2$. As we explained in Theorem 3.2, our hypothesis that $V \in C_{\mathrm{loc}}^{1, \alpha}$ implies that for $q<4 /(2-\alpha),\left(\int\left(\rho_{1, v}^{\mathrm{C}}\right)^{q}\right)^{1 / q}$ is less than some constant that depends only on $q, \alpha$ and $v=V / N$. In particular, $b$ depends only on $v$.

We see from (4.3) and (4.5) that when $V \in C_{\mathrm{loc}}^{1, \alpha}$ the power $3 / 2$ in the error term is optimal.

In order to prove (4.5) we need the following electrostatics lemma of Lieb and Yau [26]. The original version was for $\mathbf{R}^{3}$; we state it here for $\mathbf{R}^{2}$ solely for the convenience of our present application.

4.4. LEMMA (The interaction of points and densities). Given points $x_{1}, \ldots, x_{N}$ in $\mathbf{R}^{2}$, we define Voronoi cells $\Gamma_{1}, \ldots, \Gamma_{N} \subset \mathbf{R}^{2}$ by

$$
\Gamma_{j}=\left\{y \in \mathbf{R}^{2}:\left|y-x_{j}\right| \leq\left|y-x_{k}\right| \text { for all } k \neq j\right\}
$$

These $\Gamma_{j}$ have disjoint interiors and their union covers $\mathbf{R}^{2}$. We also define $R_{j}$ to be the distance from $x_{j}$ to the boundary of $\Gamma_{j}$, i.e., $R_{j}$ is half the distance of $x_{j}$ to its nearest neighbor. Let $\rho$ be any (not necessarily positive) function on $\mathbf{R}^{2}$. (In general, $\rho$ can be 
replaced by a measure, but it is not necessary for us to do so.) Then (with $D(f, g)=$ $\left.\frac{1}{2} \iint f(x) g(y)|x-y|^{-1} \mathrm{~d} x \mathrm{~d} y\right)$

$$
\begin{aligned}
\sum_{1 \leq i<j \leq N}\left|x_{i}-x_{j}\right|^{-1} \geq & -D(\rho, \rho)+\sum_{j=1}^{N} \int_{\mathbf{R}^{2}} \rho(y)\left|y-x_{j}\right|^{-1} \mathrm{~d} y \\
& +\frac{1}{8} \sum_{j=1}^{N} R_{j}^{-1}-\sum_{j=1}^{N} \int_{\Gamma_{j}} \rho(y)\left|y-x_{j}\right|^{-1} \mathrm{~d} y
\end{aligned}
$$

Proof of (4.5): We choose $\rho$ in $(4.7)$ to be the minimizer $\rho_{N, V}^{\mathrm{C}}$ for the functional $\mathcal{E}^{\mathrm{C}}$ and we choose the $x_{i}$ 's to be any (not necessarily minimizing) configuration for $\mathcal{E}^{\mathrm{P}}$. It is important, for us that a minimizer exists for $\mathcal{E}^{\mathrm{C}}$, for then $\rho$ satisfies the Euler-Lagrange equation (3.14). Since $\int \rho=N$, we conclude from (3.14) that

$$
\sum_{j=1}^{N}\left[\int \rho(y)\left|y-x_{j}\right|^{-1} \mathrm{~d} y+V\left(x_{j}\right)\right]=\sum_{j=1}^{N} V^{\mathrm{C}}\left(x_{j}\right) \geq N \mu=\int V^{\mathrm{C}} \rho=2 D(\rho, \rho)+\int V \rho
$$

Thus, if we add $\sum_{j} V\left(x_{j}\right)$ to both sides of (4.7) we have that

$$
E^{\mathrm{P}}(N, V) \geq E^{\mathrm{C}}(N, V)+\sum_{j=1}^{N} \frac{1}{8} R_{j}^{-1}-\int_{\Gamma_{j}} \rho(y)\left|y-x_{j}\right|^{-1} \mathrm{~d} y
$$

Our goal will be to control the rightmost term in (4.8) by the $R_{j}^{-1}$ term.

We split each region $\Gamma_{j}$ into two disjoint subregions, $\Gamma_{j}=A_{j} \cup B_{j}$, where

$$
A_{j}:=\left\{x:\left|x-x_{j}\right|<R_{j}\right\}, \quad B_{j}:=\left\{x \in \Gamma_{j}:\left|x-x_{j}\right| \geq R_{j}\right\} .
$$

Then, by Hölder's inequality

$$
\begin{aligned}
\sum_{j=1}^{N} \int_{B_{j}}\left|y-x_{j}\right|^{-1} \rho(y) \mathrm{d} y & \leq\left(\sum_{j} \int_{\Gamma_{j}} \rho^{3 / 2}\right)^{2 / 3}\left(\sum_{j} \int_{\left|y-x_{j}\right| \geq R_{j}}\left|y-x_{j}\right|^{-3} \mathrm{~d} y\right)^{1 / 3} \\
& =\left(\int \rho^{3 / 2}\right)^{2 / 3}\left(2 \pi \sum_{j} R_{j}^{-1}\right)^{1 / 3}
\end{aligned}
$$


If we define $X:=\sum_{j} R_{j}^{-1}$ we can rewrite (4.9) plus the $X / 8$ term in (4.8) as $\frac{1}{8} X-$ $\left(\int \rho^{3 / 2}\right)^{2 / 3} X^{1 / 3}$. The minimum of this quantity, over all values $X$, is $(4 / 3) \sqrt{2 / 3} \int \rho^{3 / 2}$, and thus we have accounted for the first error term in (4.6).

To estimate the term $I:=\sum_{j=1}^{N} \int_{A_{j}} \rho(y)\left|y-x_{j}\right|^{-1} \mathrm{~d} y$ some control is needed over the possible singularities of $\rho$. Let $p=q /(q-1)$ be the dual of $q$. Then

$$
I \leq\left(\int \rho^{q}\right)^{1 / q}\left[\sum_{j} \int_{A_{j}}\left|y-x_{j}\right|^{-p} \mathrm{~d} y\right]^{1 / p}=\left(\int \rho^{q}\right)^{1 / q}\left[\frac{2 \pi}{2-p} \sum_{j} R_{j}^{2-p}\right]^{1 / p}
$$

We note that, since $1 \leq p<2$,

$$
\left[\sum_{j=1}^{N} R_{j}^{2-p}\right]^{1 / p} \leq\left[\sum_{j=1}^{N} R_{j}^{2}\right]^{1 / p-1 / 2} N^{1 / 2}
$$

by Hölder's inequality. Now $\pi R_{j}^{2}$ is the area of the disc $A_{j}$ and thus $\pi \sum_{j} R_{j}^{2}$ is the total area of all these disjoint discs. How large can this area be? To answer this we recall Lemma A1 in the appendix which states that for the purpose of finding a set of points that minimizes the classical particle energy $\mathcal{E}^{\mathrm{P}}$ we can restrict attention to a disc of radius $R_{v}$, centered at the origin. We may therefore assume that our $x_{j}$ 's satisfy $\left|x_{j}\right| \leq R_{v}$. This we can do whether or not an energy minimizing configuration exists. Having done so and assuming that $N \geq 2$ we have that $R_{j}<R_{v}$ for all $j$, and hence all our discs are contained in a disc of radius $2 R_{v}$ centered at the origin . Thus $\sum_{j} R_{j}^{2} \leq\left(2 R_{v}\right)^{2}$, and our second error term, (4.10), is bounded above by $\left(\int \rho^{q}\right)^{1 / q}\left(\frac{2 \pi}{2-p}\right)^{1 / p}\left(2 R_{v}\right)^{-1+2 / p} N^{1 / 2}$. This yields $(4.6)$.

Q.E.D.

\section{MTF THEORY IS THE HIGH DENSITY LIMIT OF QUANTUM ME- CHANICS}

In this section we prove that the quantum energy and the quantum density are given by the corresponding MTF quantities to leading order for large $N$. These are the statements 
of Theorems 1.1 and 1.2. We shall not prove Theorem 1.2 since it follows from Theorem 1.1 in a standard way by replacing $V$ by $V+\varepsilon f$ with $f$ a function in $C_{\text {loc }}^{1, \alpha}$ and differentiating w.r.t. $\varepsilon$, see e.g., [29].

We shall prove Theorem 1.1 by giving sharp upper and lower bounds to the quantum ground state energy. The upper bound is obtained by a variational calculation using the magnetic coherent states introduced in [25,21]. The lower bound is more difficult. Besides the results of the previous sections several ingredients are needed. The first ingredient is a kinetic energy inequality of the Lieb-Thirring type [31-32], [21], [33]. Such an inequality estimates the kinetic energy of a many-body wave function from below in terms of a functional of the density. The proof of this inequality in the 2-dimensional case considered here is harder than the 3-dimensional case treated in [21] and involves some new mathematical ideas. The second ingredient is a lower bound on the exchange-correlation energy. The proof of this inequality is similar to that given in [34-35] for the 3-dimensional case.

Once the kinetic energy and exchange-correlation inequalities have been established the proof of the lower bound is completed by a coherent states analysis.

We start by discussing the magnetic coherent states used in the proofs of both the upper and lower bounds. They are constructed from the kernels

$$
\Pi_{\alpha \sigma}\left(x \sigma^{\prime}, y \sigma^{\prime \prime}\right)=\frac{B}{2 \pi} \exp \left\{i(x \times y) \cdot B-|x-y|^{2} B / 4\right\} L_{\alpha}\left(|x-y|^{2} B / 2\right) \delta_{\sigma \sigma^{\prime}} \delta_{\sigma \sigma^{\prime \prime}}
$$

of the projection operators onto the Landau levels $\alpha=0,1,2, \ldots$ with $z$-component of spin $\sigma= \pm 1 / 2$. Here $L_{\alpha}$ are Laguerre polynomials normalized by $L_{\alpha}(0)=1$. In fact, all that matters are the projectors $\Pi_{\nu}$ on the states with energy $\varepsilon_{\nu}(B)$; these are given by a sum of at most two of the projections $\Pi_{\alpha \sigma}$. More precisely,

$$
\Pi_{\nu}=\sum_{\substack{\alpha, \sigma \\ \alpha+\frac{1}{2}+\gamma \sigma=\varepsilon_{\nu}(B) / B}} \Pi_{\alpha \sigma} .
$$


We shall not need the explicit form (5.1). The three important properties of $\Pi_{\nu}$ that we use are the following

$$
\begin{aligned}
\sum_{\nu} \Pi_{\nu}\left(x \sigma^{\prime}, y \sigma^{\prime \prime}\right) & =\delta(x-y) \delta_{\sigma^{\prime} \sigma^{\prime \prime}} \\
\sum_{\sigma^{\prime}} \Pi_{\nu}\left(x \sigma^{\prime}, x \sigma^{\prime}\right) & =d_{\nu}(B) \\
H_{\mathrm{kin}} \Pi_{\nu} & =\varepsilon_{\nu}(B) \Pi_{\nu},
\end{aligned}
$$

where $H_{\text {kin }}$ is given by $(2.1)$.

Let $g$ be a real continuous function on $\mathbf{R}^{2}$, with $g(x)=0$ for $|x|>1, \int g^{2}=1$ and $\int(\nabla g)^{2}<\infty$. (The optimal choice that minimizes $\int(\nabla g)^{2}$ is the Bessel function $J_{0}$, suitably scaled and normalized.) Define $g_{r}(x)=r^{-1} g(x / r)$, with $0<r<1$ to be specified later. For each $u \in \mathbf{R}^{2}, \nu=0,1,2$, we define the operator $\Pi_{\nu u}$ - the coherent "operator" — with kernel

$$
\Pi_{\nu u}\left(x \sigma^{\prime}, y \sigma^{\prime \prime}\right)=g_{r}(x-u) \Pi_{\nu}\left(x \sigma^{\prime}, y \sigma^{\prime \prime}\right) g_{r}(y-u)
$$

It easily follows from $(5.3,4)$ and the properties of $g$ that these kernels satisfy the coherent operator identities $([36])$

$$
\begin{gathered}
\sum_{\nu} \int \Pi_{\nu u}\left(x \sigma^{\prime}, y \sigma^{\prime \prime}\right) \mathrm{d} u=\delta(x-y) \delta_{\sigma^{\prime} \sigma^{\prime \prime}} \\
\operatorname{Tr} \Pi_{\nu u}=\sum_{\sigma} \int \Pi_{\nu u}\left(x \sigma^{\prime}, x \sigma^{\prime}\right) \mathrm{d} x=d_{\nu}(B) .
\end{gathered}
$$

Moreover, a simple computation gives, using (5.5),

$$
\begin{aligned}
\operatorname{Tr}\left[H_{\mathrm{kin}} \Pi_{\nu u}\right] & =d_{\nu}(B)\left[\varepsilon_{\nu}(B)+\int\left(\nabla g_{r}\right)^{2}\right], \\
\operatorname{Tr}\left[V \Pi_{\nu u}\right] & =d_{\nu}(B) V * g_{r}^{2}(u)
\end{aligned}
$$


where $V$ is a (continuous) potential and $*$ denotes convolution. Likewise, for all $f$ with $\langle f \mid f\rangle=1$

$$
\begin{aligned}
\left\langle f\left|H_{\mathrm{kin}}\right| f\right\rangle & =\sum_{\nu} \int \varepsilon_{\nu}(B)\left\langle f\left|\Pi_{\nu u}\right| f\right\rangle \mathrm{d} u-\int\left(\nabla g_{r}\right)^{2} \\
\left\langle f\left|V * g_{r}^{2}\right| f\right\rangle & =\sum_{\nu} \int V(u)\left\langle f\left|\Pi_{\nu u}\right| f\right\rangle \mathrm{d} u .
\end{aligned}
$$

Equations $(5.9,10)$ will be used in proving the upper bound, while $(5.11,12)$ are needed for the lower bound.

\subsection{THE UPPER BOUND}

We use the variational principle of [37]. According to this principle

$$
E^{\mathrm{Q}}(N, B, V) \leq \operatorname{Tr}\left[\left(H_{\mathrm{kin}}+V\right) K\right]+\frac{1}{2} \sum_{\sigma^{\prime}, \sigma^{\prime}} \int_{\mathbf{R}^{2}} \int_{\mathbf{R}^{2}} \frac{K(x \sigma, x \sigma) K\left(y \sigma^{\prime}, y \sigma^{\prime}\right)}{|x-y|} \mathrm{d} x \mathrm{~d} y
$$

for all operators $K$, with kernel $K\left(x \sigma, y \sigma^{\prime}\right)$, satisfying

$$
0 \leq\langle f|K| f\rangle \leq\langle f \mid f\rangle
$$

for all $f$, and

$$
\operatorname{Tr}[K]=\sum_{\sigma} \int_{\mathbf{R}^{2}} K(x \sigma, x \sigma) \mathrm{d} x=N
$$

We shall choose $K$ as follows. Let $\rho^{\mathrm{MTF}}$ be the MTF density, i.e., the minimizer of the functional (1.5) with $\int \rho^{\mathrm{MTF}}=N$. Denote by $\nu_{\max }(x)$ the highest filled level. Then

$$
0 \leq \rho^{\mathrm{MTF}}(x)-\sum_{\nu \leq \nu_{\max }(x)} d_{\nu}(B)<d_{\nu_{\max }(x)+1}(B) .
$$

We introduce the filling factors

$$
f_{\nu}(x)= \begin{cases}1 & \nu \leq \nu_{\max }(x) \\ {\left[\rho^{\mathrm{MTF}}(x)-\sum_{\nu \leq \nu_{\max }(x)} d_{\nu}(B)\right] / d_{\nu_{\max }(x)+1}(B),} & \nu=\nu_{\max }(x)+1 \\ 0 & \nu>\nu_{\max }(x)+1\end{cases}
$$


and define

$$
K\left(x \sigma, y \sigma^{\prime}\right)=\sum_{\nu} \int f_{\nu}(u) \Pi_{\nu u}\left(x \sigma, y \sigma^{\prime}\right) d u,
$$

with $\Pi_{\nu u}$ as in (5.6). It follows from (5.7) that $K$ satisfies (5.14) and from (5.8), (5.16) and (5.17) that $\operatorname{Tr}[K]=\int \rho^{\mathrm{MTF}}(u) \mathrm{d} u=N$.

Note that (5.4) and (5.6) imply

$$
\sum_{\sigma} K(x \sigma, x \sigma)=\rho^{\mathrm{MTF}} * g_{r}^{2}(x)
$$

Hence, the last term in (5.13) is $D\left(\rho^{\mathrm{MTF}} * g_{r}^{2}, \rho^{\mathrm{MTF}} * g_{r}^{2}\right)$, where the functional $D$ was defined in (1.4). By convexity of $D$ we find that

$$
D\left(\rho^{\mathrm{MTF}} * g_{r}^{2}, \rho^{\mathrm{MTF}} * g_{r}^{2}\right) \leq D\left(\rho^{\mathrm{MTF}}, \rho^{\mathrm{MTF}}\right) .
$$

From $(5.9),(5.10)$ and (5.13) we obtain

$$
\begin{aligned}
E^{\mathrm{Q}}(N, B, V) & \leq \mathcal{E}^{\mathrm{MTF}}\left(\rho^{\mathrm{MTF}}\right)+N \int\left(\nabla g_{r}\right)^{2} \mathrm{~d} x+\int\left[V * g_{r}^{2}(*)-V(x)\right] \rho^{\mathrm{MTF}}(x) \mathrm{d} x \\
& \leq E^{\mathrm{MTF}}(N, V, B)+N r^{-2} \int(\nabla g(x))^{2} \mathrm{~d} x+N^{2} \sup _{|x|<R}\left[v * g_{r}^{2}(x)-v(x)\right],(5
\end{aligned}
$$

where $R=R_{v}$ is the finite radius and we have written $V=N v$. Since $v$ is in $C^{1, \alpha}$

$$
\sup _{|x|<R}\left|v * g_{r}^{2}(x)-v(x)\right| \leq \text { (const.) } r
$$

We can choose $r=r_{N}$ such that $r_{N} \rightarrow 0$ and $r_{N}^{-2} / N \rightarrow 0$ as $N \rightarrow \infty$. This means that $r_{N}$ should go to zero but still be large compared with the average spacing $N^{-1 / 2}$ between electrons. The optimal choice is of the order $r=$ (const.) $N^{-1 / 3}$. Thus the error

$$
\left[E^{\mathrm{Q}}(N, B, V)-E^{\mathrm{MTF}}(N, B, V)\right] N^{-2}
$$

is bounded above by a function $\varepsilon_{N}^{+}(v)=c^{+}(v) N^{-1 / 3}$ (independent of $B$ ). This finishes the proof of the upper bound. 


\subsection{THEOREM (Lieb-Thirring inequality in 2 dimensions).}

Let $H_{\mathbf{A}}=\frac{1}{2}(i \nabla-\mathbf{A})^{2}+\mathbf{S} \cdot \mathbf{B}$. (This is the operator $H_{\mathrm{kin}}$ from (2.1) with $\gamma=1$.) Let $W$ be a locally integrable function and denote by $e_{1}(W), e_{2}(W), \ldots$ the negative eigenvalues (if any) of the operator $H=H_{\mathbf{A}}-W$ defined on $L^{2}\left(\mathbf{R}^{2} ; \mathbf{C}^{2}\right)$, the space of wave functions of a single spin- $\frac{1}{2}$ particle. Define $|W|_{+}(x)=\frac{1}{2}[|W(x)|+W(x)]$. For all $0<\lambda<1$ we then have the estimate

$$
\sum_{j}\left|e_{j}(W)\right| \leq \lambda^{-1} \frac{B}{2 \pi} \int_{\mathbf{R}^{2}}|W|_{+}(x) \mathrm{d} x+\frac{3}{4}(1-\lambda)^{-2} \int_{\mathbf{R}^{2}}|W|_{+}^{2}(x) \mathrm{d} x .
$$

Proof: For any self-adjoint operator $A$ we denote by $N_{\alpha}(A)$ the number of eigenvalues of $A$ greater than or equal to $\alpha$.

Since replacing $W$ by its positive part $|W|_{+}$will only enhance the sum of the negative eigenvalues we shall henceforth assume that $W$ is positive, i.e., $W=|W|_{+}$. We consider the Birman-Schwinger kernel

$$
K_{E}=W^{1 / 2}\left(H_{\mathbf{A}}+E\right)^{-1} W^{1 / 2} .
$$

According to the Birman-Schwinger principle (see, e.g., [38], p.89) the number $N_{E}(-H)$ of eigenvalues of $H$ below $-E$ is equal to the number $N_{1}\left(K_{E}\right)$ of eigenvalues of $K_{E}$ greater than or equal to 1 . We find

$$
\sum_{j}\left|e_{j}(W)\right|=\int_{0}^{\infty} N_{E}(-H) \mathrm{d} E=\int_{0}^{\infty} N_{1}\left(K_{E}\right) \mathrm{d} E
$$

In order to estimate $N_{1}\left(K_{E}\right)$ we decompose the Birman-Schwinger kernel into a part $K_{E}^{0}$ coming from the lowest Landau level and a part $K_{E}^{>}$coming from the higher levels. If $\Pi_{0}$ is the projection onto the lowest Landau band these two parts are defined by

$$
K_{E}^{0}=W^{1 / 2} \Pi_{0}\left(H_{\mathbf{A}}+E\right)^{-1} \Pi_{0} W^{1 / 2}=E^{-1} W^{1 / 2} \Pi_{0} W^{1 / 2}
$$


and

$$
K_{E}^{>}=W^{1 / 2}\left(I-\Pi_{0}\right)\left(H_{\mathbf{A}}+E\right)^{-1}\left(I-\Pi_{0}\right) W^{1 / 2} .
$$

Since $\Pi_{0}$ commutes with $H_{\mathrm{A}}$ we have $K_{E}=K_{E}^{0}+K_{E}^{>}$.

Now we use Fan's theorem [39], which states that if $\mu_{1}(X) \geq \mu_{2}(X) \geq \ldots$ denote the eigenvectors of a self-adjoint compact operator $X$ then $\mu_{n+m+1}(X+Y) \leq \mu_{n+1}(X)+$ $\mu_{m+1}(Y)$ for $n, m \geq 0$. From this we have $N_{1}(X+Y) \leq N_{\lambda}(X)+N_{1-\lambda}(Y)$ which, in our case, reads as follows.

$$
N_{1}\left(K_{E}\right) \leq N_{\lambda}\left(K_{E}^{0}\right)+N_{1-\lambda}\left(K_{E}^{>}\right), \text {for } 0 \leq \lambda \leq 1
$$

This inequality permits us to consider the two parts of $K_{E}$ separately. We first consider the contribution from the lowest level: $N_{\lambda}\left(K_{E}^{0}\right)=N_{\lambda E}\left(W^{1 / 2} \Pi_{0} W^{1 / 2}\right)$. We get

$$
\begin{aligned}
\int_{0}^{\infty} N_{\lambda}\left(K_{E}^{0}\right) \mathrm{d} E & =\int_{0}^{\infty} N_{\lambda E}\left(W^{1 / 2} \Pi_{0} W^{1 / 2}\right) \mathrm{d} E \\
& =\lambda^{-1} \int_{0}^{\infty} N_{E}\left(W^{1 / 2} \Pi_{0} W^{1 / 2}\right) \mathrm{d} E=\lambda^{-1} \operatorname{Tr}\left(W^{1 / 2} \Pi_{0} W^{1 / 2}\right) \\
& =\lambda^{-1} \int \Pi_{0}(x, x) W(x) \mathrm{d} x=\lambda^{-1} \frac{B}{2 \pi} \int W(x) \mathrm{d} x
\end{aligned}
$$

The second part is straightforward. We first notice that $H_{\mathbf{A}}\left(I-\Pi_{0}\right) \geq B\left(I-\Pi_{0}\right)$. Hence $H_{\mathbf{A}}\left(I-\Pi_{0}\right) \geq \frac{2}{3}\left(H_{\mathbf{A}}+\frac{1}{2} B\right)\left(I-\Pi_{0}\right) \geq \frac{1}{3}(i \nabla-\mathbf{A})^{2}\left(I-\Pi_{0}\right)$. (Note that $(i \nabla-\mathbf{A})^{2}$ commutes with $\Pi_{0}$.) Since the operator inequality $0<X \leq Y$ implies $X^{-1} \leq Y^{-1}$ we have that

$$
K_{E}^{>} \leq W^{1 / 2}\left(I-\Pi_{0}\right)\left[\frac{1}{3}(i \nabla-\mathbf{A})^{2}+E\right]^{-1}\left(I-\Pi_{0}\right) W^{1 / 2} \leq W^{1 / 2}\left[\frac{1}{3}(i \nabla-\mathbf{A})^{2}+E\right]^{-1} W^{1 / 2} .
$$

We conclude that $N_{1-\lambda}\left(K_{E}^{>}\right) \leq N_{1}\left(\widetilde{K}_{E}\right)$, where

$$
\widetilde{K}_{E}=\left((1-\lambda)^{-1} W\right)^{1 / 2}\left[\frac{1}{3}(i \nabla-\mathbf{A})^{2}+E\right]^{-1}\left((1-\lambda)^{-1} W\right)^{1 / 2}
$$


is the Birman-Schwinger kernel for the operator $\widetilde{H}=\frac{1}{3}\left[(i \nabla-\mathbf{A})^{2}-3(1-\lambda)^{-1} W\right]$. The Birman-Schwinger principle implies that $\int_{0}^{\infty} N_{1}\left(\widetilde{K}_{E}\right) \mathrm{d} E$ is the sum of the negative eigenvalues of $\widetilde{H}$. An estimate on this quantity follows from the standard Lieb-Thirring inequality, i.e.,

$$
\int_{0}^{\infty} N_{1-\lambda}\left(K_{E}^{>}\right) \mathrm{d} E \leq(0.24) \frac{1}{3} \int\left(\frac{3}{(1-\lambda)} W(x)\right)^{2} \mathrm{~d} x \leq \frac{3}{4}(1-\lambda)^{-2} \int W(x)^{2} \mathrm{~d} x
$$

The constant 0.24 can be found as $L_{1,2}$ in [40], Eq. (51). It was improved slightly by Blanchard and Stubbe [41], see also [42]. In these references only the case $\mathbf{A}=0$ was considered. It is, however, a simple consequence of the diamagnetic inequality (see [43]) that the constant is independent of $\mathbf{A}$.

Q.E.D.

The Lieb-Thirring inequality in Theorem 5.2 implies an estimate on the kinetic energy

$$
T_{\psi}=\left\langle\psi\left|\sum_{j=1}^{N} H_{\mathrm{kin}}^{(j)}\right| \psi\right\rangle
$$

in terms of the one-particle density

$$
\rho_{\psi}(x)=N \sum_{\sigma_{1}= \pm 1 / 2} \ldots \sum_{\sigma_{N}= \pm 1 / 2} \int_{\mathbf{R}^{2(N-1)}}\left|\psi\left(x, x_{2} \ldots, x_{N} ; \sigma_{1}, \ldots, \sigma_{N}\right)\right|^{2} \mathrm{~d} x_{2} \ldots \mathrm{d} x_{N}
$$

Here $\psi$ is a normalized $N$ particle fermionic wave function.

5.3 COROLLARY (Kinetic energy inequality in 2 dimensions). Let $T_{\psi}$ and $\rho_{\psi}$ be defined as above. Then for all $0<\lambda<1$ we have

$$
T_{\psi} \geq\left\{\begin{array}{ll}
0, & \text { if } \rho_{\psi} \leq \lambda^{-1} \frac{B}{\pi} \\
\frac{1}{3}(1-\lambda)^{2} \int\left[\rho_{\psi}(x)-\lambda^{-1} \frac{B}{\pi}\right]^{2} \mathrm{~d} x, & \text { if } \rho_{\psi} \geq \lambda^{-1} \frac{B}{\pi}
\end{array} .\right.
$$

Proof: The inequality in Theorem 5.2 holds for the operator $H_{\text {kin }}-W$ if $|\gamma| \geq 1$. If $|\gamma|<1$, however, one should choose $\Pi_{0}$ in the proof of Theorem 5.2 as the projection onto the levels $\nu=0$ and $\nu=1$ (not only onto $\nu=0$ ). Equation (5.21) is then no 
longer an identity but a bound. In this way one concludes that the negative eigenvalues $e_{1}(W), e_{2}(W), \ldots$ for $H_{\text {kin }}-W$ satisfy

$$
\sum_{j}\left|e_{j}(W)\right| \leq \alpha \int_{\mathbf{R}^{2}}|W|_{+}(x) \mathrm{d} x+\beta \int_{\mathbf{R}^{2}}|W|_{+}^{2}(x) \mathrm{d} x
$$

with $\alpha=\lambda^{-1} \frac{B}{\pi}$ and $\beta=\frac{3}{4}(1-\lambda)^{-2}$. This bound is clearly valid for all $\gamma$.

The proof of (5.22) now follows by a standard Legendre transformation. In fact, if $W \geq 0$ we have

$$
T_{\psi}=\left\langle\psi\left|\sum_{j} H_{\mathrm{kin}}^{(j)}-W\left(x_{j}\right)\right| \psi\right\rangle+\int W \rho_{\psi} \geq \int_{\mathbf{R}^{2}}\left[W \rho_{\psi}-\alpha W-\beta W^{2}\right]
$$

Since the Legendre transformation of the function $W \mapsto \alpha W+\beta W^{2}$ is the function

$$
\rho \mapsto \sup _{W \geq 0}\left[\rho W-\alpha W-\beta W^{2}\right]=\left\{\begin{array}{ll}
0, & \text { if } \rho \leq \alpha \\
(4 \beta)^{-1}(\rho-\alpha)^{2}, & \text { if } \rho>\alpha
\end{array},\right.
$$

we see that (5.22) follows by making the optimal choice for $W$ in (5.23).

Q.E.D.

5.4 LEMMA (Exchange inequality in 2 dimensions). Let $\psi \in \bigotimes^{N} L^{2}\left(\mathbf{R}^{2} ; \mathbf{C}^{2}\right)$ be any normalized $N$ particle wave-function (not necessarily fermionic) and let

$\rho_{\psi}(x)=\sum_{i=1}^{N} \sum_{\sigma_{1}= \pm 1 / 2} \ldots \sum_{\sigma_{N}= \pm 1 / 2} \int_{\mathbf{R}^{2 N}}\left|\psi\left(x_{1}, \ldots, x_{N} ; \sigma_{1}, \ldots, \sigma_{N}\right)\right|^{2} \mathrm{~d} x_{1} \ldots \mathrm{d} x_{i-1} \mathrm{~d} x_{i+1} \ldots \mathrm{d} x_{N}$ be the corresponding one-particle density. Then

$$
\begin{aligned}
\sum_{\sigma_{1}} \ldots \sum_{\sigma_{N}} \int_{\mathbf{R}^{2 N}}|\psi|^{2} \sum_{1 \leq i<j \leq N}\left|x_{i}-x_{j}\right|^{-1} \mathrm{~d} x_{1} \ldots \mathrm{d} x_{N} \\
\quad \geq \frac{1}{2} \int_{\mathbf{R}^{2}} \int_{\mathbf{R}^{2}} \rho_{\psi}(x) \rho_{\psi}(y)|x-y|^{-1} \mathrm{~d} x \mathrm{~d} y-192(2 \pi)^{1 / 2} \int_{\mathbf{R}^{2}} \rho_{\psi}(x)^{3 / 2} \mathrm{~d} x .
\end{aligned}
$$

Proof: The proof is essentially the same as in [34], where the three dimensional equivalent of (1.1) was proved. Our presentation is inspired by [44]. 
We use the representation (in 3 dimensions a similar representation was originally used by Fefferman and de La Llave [45])

$$
|x-y|^{-1}=\pi^{-1} \int_{\mathbf{R}^{2}} \int_{\mathbf{R}} \chi_{R}(x-z) \chi_{R}(y-z) R^{-4} \mathrm{~d} R \mathrm{~d} z,
$$

where $\chi_{R}$ is the characteristic function of the ball of radius $R$ centered at the origin. If we use (5.25) to represent $\sum_{i<j}\left|x_{i}-x_{j}\right|^{-1}$ we can estimate the integrand as follows.

$$
\begin{aligned}
\sum_{1 \leq i<j \leq N} \chi_{R}\left(x_{i}-z\right) \chi_{R}\left(x_{j}-z\right)=\frac{1}{2}\left(\sum_{i} \chi_{R}\left(x_{i}-z\right)\right)^{2}-\frac{1}{2} \sum_{i} \chi_{R}\left(x_{i}-z\right) \\
\quad=\frac{1}{2}\left(\sum_{i} \chi_{R}\left(x_{i}-z\right)-\int \rho_{\psi}(y) \chi_{R}(y-z) \mathrm{d} y\right)^{2}+\sum_{i} \chi_{R}\left(x_{i}-z\right) \int \rho_{\psi}(z) \chi_{R}(y-z) \mathrm{d} y \\
\quad-\frac{1}{2}\left(\int \rho_{\psi}(y) \chi_{R}(y-z) \mathrm{d} y\right)^{2}-\frac{1}{2} \sum_{i} \chi_{R}\left(x_{i}-z\right) \\
\quad \geq \sum_{i} \chi_{R}\left(x_{i}-z\right) \int \rho_{\psi}(y) \chi_{R}(y-z) \mathrm{d} y-\frac{1}{2}\left(\int \rho_{\psi}(y) \chi_{R}(y-z) \mathrm{d} y\right)^{2}-\frac{1}{2} \sum_{i} \chi_{R}\left(x_{i}-z\right) .
\end{aligned}
$$

If we integrate this inequality over the measure $R^{-4} \mathrm{~d} R \mathrm{~d} z$, the last term, $\frac{1}{2} \sum_{i} \chi_{R}\left(x_{i}-z\right)$, will give a divergent integral. For the purpose of a lower bound, however, we can restrict the integration in (5.25) to $R>r(z)$, where $r(z)>0$ is some specific function we shall choose below. Using the fact that

$$
\sum_{\sigma_{1}} \ldots \sum_{\sigma_{N}} \int_{\mathbf{R}^{2 N}}|\psi|^{2} \sum_{i} \chi_{R}\left(x_{i}-z\right) \mathrm{d} x_{1} \ldots \mathrm{d} x_{N}=\int_{\mathbf{R}^{2}} \rho_{\psi}(y) \chi_{R}(y-z) \mathrm{d} y
$$

we obtain,

$$
\begin{aligned}
& \sum_{\sigma_{1}} \ldots \sum_{\sigma_{N}} \int_{\mathbf{R}^{2 N}}|\psi|^{2} \sum_{i<j}\left|x_{i}-x_{j}\right|^{-1} \mathrm{~d} x_{1} \ldots \mathrm{d} x_{N} \\
& \geq \frac{1}{2} \pi^{-1} \int_{\mathbf{R}^{2}} \int_{R>r(z)}\left(\int \rho_{\psi}(y) \chi_{R}(y-z) \mathrm{d} y\right)^{2} R^{-4} \mathrm{~d} R \mathrm{~d} z
\end{aligned}
$$




$$
\begin{aligned}
& -\frac{1}{2} \pi^{-1} \int_{\mathbf{R}^{2}} \int_{R>r(z)} \int \rho_{\psi}(y) \chi_{R}(y-z) \mathrm{d} y R^{-4} \mathrm{~d} R \mathrm{~d} z \\
& \geq \frac{1}{2} \pi^{-1} \iint \rho_{\psi}(x) \rho_{\psi}(y)|x-y|^{-1} \mathrm{~d} x \mathrm{~d} y \\
& -\frac{1}{2} \pi^{-1} \int_{\mathbf{R}^{2}} \int_{R<r(z)} \pi^{2} \rho_{\psi}^{*}(z)^{2} \mathrm{~d} R \mathrm{~d} z-\frac{1}{2} \pi^{-1} \int_{\mathbf{R}^{2}} \int_{R>r(z)} R^{-2} \pi \rho_{\psi}^{*}(z) \mathrm{d} R \mathrm{~d} z .
\end{aligned}
$$

Here we have introduced the Hardy-Littlewood maximal function

$$
\rho_{\psi}^{*}(z)=\sup _{R}\left(\pi R^{2}\right)^{-1} \int \rho_{\psi}(y) \chi_{R}(y-z) \mathrm{d} y
$$

which, viewed as a map from $L^{p}\left(\mathbf{R}^{2}\right)$ to $L^{p}\left(\mathbf{R}^{2}\right)$, is a bounded map for all $p>1$ (see [46], pp. 54-58). The error terms in (5.26) can be computed as

$$
\begin{gathered}
\frac{1}{2} \pi^{-1} \int_{\mathbf{R}^{2}}\left[\int_{R<r(z)} \pi^{2} \rho_{\psi}^{*}(z)^{2} \mathrm{~d} R+\int_{R>r(z)} R^{-2} \pi \rho_{\psi}^{*}(z) \mathrm{d} R\right] \mathrm{d} z \\
=\frac{1}{2} \pi^{-1} \int_{\mathbf{R}^{2}}\left(r(z) \pi^{2} \rho_{\psi}^{*}(z)^{2}+r(z)^{-1} \pi \rho_{\psi}^{*}(z)\right) \mathrm{d} z .
\end{gathered}
$$

The optimal choice for $r(z)$ is $r(z)=\left(\pi \rho^{*}(z)\right)^{-1 / 2}$. This means that the error is $\pi^{1 / 2} \int_{\mathbf{R}^{2}} \rho_{\psi}^{*}(z)^{3 / 2} \mathrm{~d} z$, but this can be estimated by the maximal inequality to be less than $192(2 \pi)^{1 / 2} \int_{\mathbf{R}^{2}} \rho(z)^{3 / 2} \mathrm{~d} z$. Q.E.D.

\subsection{THE LOWER BOUND}

Our goal here is to give a lower bound to $E^{\mathrm{Q}}(N, B, V)$ in terms of $E^{\mathrm{MTF}}(N, B, V)$ with errors of lower order than $N^{2}$ as $N$ tends to infinity. It is important here that $V=N v$, where $v$ is fixed. To be more precise we shall prove that

$$
N^{-2}\left[E^{\mathrm{Q}}(N, B, V)-E^{\mathrm{MTF}}(N, B, V)\right] \geq-\varepsilon_{N}^{-}(v),
$$

where $\varepsilon_{N}^{-}(v)$ is a non-negative function which tends to 0 as $N \rightarrow \infty$ for fixed $v$. Note, however, that $\varepsilon_{N}^{-}(v)$ does not depend on $B$. 
We shall treat the cases of large $B$ and small $B$ separately. In the large $B$ regime we prove (5.27) by a comparison with the classical models discussed in Sects. III and IV. In the small $B$ regime we use magnetic coherent states, Theorem 5.2 and Lemma 5.4.

The dividing line between large and small $B$ is determined as follows. If the minimizer $\rho_{1, v}^{\mathrm{C}}$ of $\mathcal{E}^{\mathrm{C}}$, with $\int \rho_{1, v}^{\mathrm{C}}=1$ and confining potential $v$, is bounded (e.g. for $v(x)=|x|^{2}$ ) then we define small $B$ to mean

$$
B / N \leq \beta_{c}:=2 \pi \sup _{x} \rho_{1, v}^{\mathrm{C}}(x)
$$

As explained in (3.20) we have for $\beta \geq \beta_{c}$ that $E^{\mathrm{MTF}}(1, \beta, v)=E^{\mathrm{C}}(1, v)$.

For the general class of $v$ where we do not know that the minimizer $\rho_{1, v}^{\mathrm{C}}$ is bounded we simply define

$$
\beta_{c}=N^{1 / 3}
$$

By Theorem 3.4 we then have that the function

$$
\delta(N, v)=\sup _{\beta \geq \beta_{c}}\left|E^{\mathrm{MTF}}(1, \beta, v)-E^{\mathrm{C}}(1, v)\right|
$$

tends to zero as $N$ tends to infinity.

Case 1, $B / N \geq \beta_{c}$ : By simply ignoring the kinetic energy operator, which we had normalized to be positive, we have the obvious inequality $E^{\mathrm{Q}}(N, B, V) \geq E^{\mathrm{P}}(N, V)$ where $E^{\mathrm{P}}$ is the energy of the classical point problem.

From Lemma 4.3 we can therefore conclude that

$$
E^{\mathrm{Q}}(N, B, V) \geq E^{\mathrm{P}}(N, V) \geq E^{\mathrm{C}}(N, V)-b(v) N^{3 / 2}
$$

Since $E^{\mathrm{C}}(N, V)=N^{2} E^{\mathrm{C}}(1, v)$ and $E^{\mathrm{MTF}}(N, B, V)=N^{2} E^{\mathrm{MTF}}(1, B / N, v)$ we have from (5.29) that 


$$
E^{\mathrm{Q}}(N, B, V) \geq E^{\mathrm{MTF}}(N, B, V)-\delta(N, v) N^{2}-b(v) N^{3 / 2}
$$

Thus (5.27) holds with $\varepsilon_{N}^{-}(v)=\delta(N, v)+b(v) N^{-1 / 2}$.

We emphasize again that if $\rho_{1, v}^{\mathrm{C}}$ is bounded (e.g. for $v=k|x|^{2}$ ) then $\delta(N, v)$ is not needed.

Case 2, $B / N \leq \beta_{c}$ : In this case we use inequality (5.24) to reduce the many-body problem to a one-body problem.

Let $\psi$ be the many-body ground state* for $H_{N}$. The correlation estimate (5.24) gives

$$
E^{\mathrm{Q}}(N, B, V)=\left\langle\psi\left|H_{N}\right| \psi\right\rangle \geq \sum_{j=1}^{N}\left\langle\psi\left|H_{\mathrm{kin}}^{(j)}+V\left(x_{j}\right)\right| \psi\right\rangle+D\left(\rho_{\psi}, \rho_{\psi}\right)-C \int_{\mathbf{R}^{2}} \rho_{\psi}^{3 / 2}(x) \mathrm{d} x
$$

We first estimate the last term in (5.32) in terms of the kinetic energy $T_{\psi}=\left\langle\psi\left|\sum_{j=1}^{N} H_{\mathrm{kin}}^{(j)}\right| \psi\right\rangle$ of $\psi$. According to (5.22) we have

$$
\begin{aligned}
\int \rho_{\psi}^{3 / 2} & \leq \text { (const.) } B^{1 / 2} \int_{\left.\rho_{\psi}<\text { (const. }\right) B} \rho_{\psi}+(\text { const. })\left(\int \rho_{\psi}\right)^{1 / 2}\left(\int_{\rho_{\psi} \geq \text { (const.) } B} \rho_{\psi}^{2}\right)^{1 / 2} \\
& \leq \text { (const. })\left(\sqrt{\beta_{c}} N^{3 / 2}+\sqrt{T_{\psi}} N^{1 / 2}\right) .
\end{aligned}
$$

Hence, for all $0<\varepsilon<1$ (we shall later choose $\varepsilon \sim N^{-1 / 2}$ ) we have

$E^{\mathrm{Q}}(N, B, V) \geq \sum_{j=1}^{N}\left\langle\psi\left|(1-\varepsilon) H_{\mathrm{kin}}^{(j)}+V\left(x_{j}\right)\right| \psi\right\rangle+D\left(\rho_{\psi}, \rho_{\psi}\right)-($ const. $)\left(\sqrt{\beta_{c}} N^{3 / 2}+\varepsilon^{-1} N\right)$.

where we have used that

$$
\varepsilon T_{\psi}-\text { (const.) } \sqrt{T_{\psi}} N^{1 / 2} \geq- \text { (const.) } \varepsilon^{-1} N \text {. }
$$

\footnotetext{
* Since the exterior potential $V$ tends to infinity at infinity $H_{N}$ will have a ground state.
} 
To relate (5.34) to the MTF problem we use the inequality

$0 \leq D\left(\rho_{\psi}-\rho^{\mathrm{MTF}}, \rho_{\psi}-\rho^{\mathrm{MTF}}\right)=-\left\langle\left.\psi\left|\sum_{j=1}^{N} \rho^{\mathrm{MTF}} *\right| x_{j}\right|^{-1} \mid \psi\right\rangle+D\left(\rho_{\psi}, \rho_{\psi}\right)+D\left(\rho^{\mathrm{MTF}}, \rho^{\mathrm{MTF}}\right)$,

which is a consequence of the positive definiteness of the kernel $|x-y|^{-1}$. Inserting this in $(5.34)$ gives

$$
\begin{aligned}
E^{\mathrm{Q}}(N, B, V) & \geq \sum_{j=1}^{N}\left\langle\left.\psi\left|(1-\varepsilon) H_{\mathrm{kin}}^{(j)}+V\left(x_{j}\right)+\rho^{\mathrm{MTF}} *\right| x_{j}\right|^{-1} \mid \psi\right\rangle \\
& -D\left(\rho^{\mathrm{MTF}}, \rho^{\mathrm{MTF}}\right)-(\text { const. })\left(\sqrt{\beta_{c}} N^{3 / 2}+\varepsilon^{-1} N\right) .
\end{aligned}
$$

Since we have normalized the potential to be positive we have that $(1-\varepsilon)^{-1} V(x) \geq V(x)$ and also $(1-\varepsilon)^{-1} \rho^{\mathrm{MTF}} *|x|^{-1} \geq \rho^{\mathrm{MTF}} *|x|^{-1}$.

Hence

$$
\begin{aligned}
E^{\mathrm{Q}}(N, B, V) & \geq(1-\varepsilon)\left\langle\psi\left|\sum_{j=1}^{N}\left(H_{\text {kin }}^{(j)}+V\left(x_{j}\right)+\rho^{\mathrm{MTF}} *\left|x_{j}\right|^{-1}\right)\right| \psi\right\rangle \\
& -D\left(\rho^{\mathrm{MTF}}, \rho^{\mathrm{MTF}}\right)-(\text { const. })\left(\sqrt{\beta_{c}} N^{3 / 2}+\varepsilon^{-1} N\right) .
\end{aligned}
$$

Obviously

$$
\left\langle\left.\psi\left|\sum_{j=1}^{N}\left(H_{\mathrm{kin}}^{(j)}+V\left(x_{j}\right)\right)+\rho^{\mathrm{MTF}} *\right| x_{j}\right|^{-1} \mid \psi\right\rangle \geq \sum_{j=1}^{N} e_{j},
$$

where $e_{1}, e_{2}, \ldots, e_{N}$ are the $N$ lowest eigenvalues of the one-particle Hamiltonian

$$
H_{1}^{\mathrm{MTF}}=H_{\mathrm{kin}}+V(x)+\rho^{\mathrm{MTF}} *|x|^{-1}=H_{\mathrm{kin}}+V^{\mathrm{MTF}}(x)
$$

We shall estimate $\sum_{j=1}^{N} e_{j}$ by a straightforward coherent states analysis.

Let $f_{1}, \ldots, f_{N}$ be the $N$ lowest normalized eigenfunctions of $H_{1}^{\mathrm{MTF}}$. For technical reasons we introduce a modified operator $\widetilde{H}_{1}^{\mathrm{MTF}}$ which is obtained from $H_{1}^{\mathrm{MTF}}$ by replacing $V^{\mathrm{MTF}}$ by the truncated potential

$$
\widetilde{V}^{\mathrm{MTF}}(x)=\left\{\begin{array}{ll}
V^{\mathrm{MTF}}(x) & |x| \leq R_{v} \\
C N & |x| \geq R_{v}
\end{array},\right.
$$


where $R_{v}$ is the finite radius given in the appendix and $C=\inf _{|x|>R_{v}} V^{\mathrm{MTF}}(x) / N$ is independent of $N$ by the scaling (2.9) of MTF theory. Note that $V^{\mathrm{MTF}} \geq \widetilde{V}^{\mathrm{MTF}}$. Then from $(5.11,12)$ we have

$$
\begin{aligned}
\sum_{j=1}^{N} e_{j} & =\sum_{j=1}^{N}\left\langle f_{j}\left|H_{1}^{\mathrm{MTF}}\right| f_{j}\right\rangle \geq \sum_{j=1}^{N}\left\langle f_{j}\left|\widetilde{H}_{1}^{\mathrm{MTF}}\right| f_{j}\right\rangle \\
& =\sum_{\nu} \int \mathrm{d} u\left(\varepsilon_{\nu}(B)+\widetilde{V}^{\mathrm{MTF}}(u)\right) \sum_{j=1}^{N}\left\langle f_{j}\left|\Pi_{\nu u}\right| f_{j}\right\rangle \\
& -r^{-2} N \int(\nabla g)^{2}+\sum_{j=1}^{N}\left\langle f_{j}\left|\widetilde{V}^{\mathrm{MTF}}-\widetilde{V}^{\mathrm{MTF}} * g_{r}^{2}\right| f_{j}\right\rangle .
\end{aligned}
$$

We first consider the last term. Writing $\sum_{j=1}^{N}\left|f_{j}(x)\right|^{2}=\widetilde{\rho}(x)$ we have

$$
\begin{gathered}
\sum_{j=1}^{N}\left\langle f_{j}\left|\widetilde{V}^{\mathrm{MTF}}-\widetilde{V}^{\mathrm{MTF}} * g_{r}^{2}(x)\right| f_{j}\right\rangle=\int_{|x|<R_{v}+r}\left[\widetilde{V}^{\mathrm{MTF}}(x)-\widetilde{V}^{\mathrm{MTF}} * g_{r}^{2}(x)\right] \widetilde{\rho}(x) \mathrm{d} x \\
\quad \geq \int_{|x| \leq R_{v}-r}\left[V^{\mathrm{MTF}}(x)-V^{\mathrm{MTF}} * g_{r}^{2}(x)\right] \widetilde{\rho}(x) \mathrm{d} x-\int_{R_{v}-r \leq|x| \leq R_{v}+r} \widetilde{V}^{\mathrm{MTF}} * g_{r}^{2}(x) \widetilde{\rho}(x) \mathrm{d} x .
\end{gathered}
$$

Since $V^{\mathrm{MTF}}=N v^{\mathrm{MTF}}$ and $\int \widetilde{\rho}=N$ we have

$$
\sum_{j=1}^{N}\left\langle f_{j}\left|\widetilde{V}^{\mathrm{MTF}}-\widetilde{V}^{\mathrm{MTF}} * g_{r}^{2}\right| f_{j}\right\rangle \geq-N^{2} \sup _{|x|<R_{v}}\left|v^{\mathrm{MTF}}(x)-v^{\mathrm{MTF}} * g_{r}^{2}(x)\right|-\left(\sup _{|x|<R_{v}+r} v^{\mathrm{MTF}}(x)\right) R_{v}^{2} r N^{2} .
$$

We can then write $(5.41)$ as

$$
\sum_{j=1}^{N} e_{j} \geq \sum_{\nu} \int\left(\varepsilon_{\nu}(B)+\widetilde{V}^{\mathrm{MTF}}(u)\right) \sum_{j=1}^{N}\left\langle f_{j}\left|\Pi_{\nu u}\right| f_{j}\right\rangle \mathrm{d} u-N^{2} \widetilde{\varepsilon}_{N}(v)
$$

where

$$
\begin{aligned}
\widetilde{\varepsilon}_{N}(v) & =\sup _{|x|<R_{v}}\left|v^{\mathrm{MTF}}(x)-v^{\mathrm{MTF}} * g_{r}^{2}(x)\right| \\
& +\left(\sup _{|x|<R_{v}+r} v^{\mathrm{MTF}}(x)\right) R_{v}^{2} r-N^{-1} r^{2} \int(\nabla g)^{2} \\
& \leq C(v) N^{-1 / 3} .
\end{aligned}
$$


For the last step we made the choice $r \sim N^{-1 / 3}$.

We focus next on the first term in (5.43). It has the form

$$
\int\left(\varepsilon_{\nu}(B)+\widetilde{V}^{\mathrm{MTF}}(u)\right) \rho_{\nu}(u) \mathrm{d} u
$$

where we have denoted $\sum_{j=1}^{N}\left\langle f_{j}\left|\Pi_{\nu u}\right| f_{j}\right\rangle$ by $\rho_{\nu}(u)$. These functions satisfy

$$
0 \leq \rho_{\nu}(u) \leq \operatorname{Tr} \Pi_{\nu u}=d_{\nu}(B)
$$

and

$$
\sum_{\nu} \int \rho_{\nu}(u) \mathrm{d} u=N
$$

We obtain a lower bound to $(5.45)$ by minimizing over all functions $\rho_{\nu}$ satisfying (5.46) and (5.47).

Minimizers $\rho_{\nu}$ can be constructed as follows. There is a $\mu>0$ such that

$$
\rho_{\nu}(u)= \begin{cases}d_{\nu}(B) & \text { if } \varepsilon_{\nu}(B)+\widetilde{V}^{\mathrm{MTF}}(u)<\mu \\ 0 & \text { if } \varepsilon_{\nu}(B)+\widetilde{V}^{\mathrm{MTF}}(u)>\mu \\ \leq d_{\nu}(B) & \text { if } \varepsilon_{\nu}(B)+\widetilde{V}^{\mathrm{MTF}}(u)=\mu\end{cases}
$$

All families $\rho_{\nu}$ satisfying (5.48) and the constraint (5.47) are minimizers. Note that it is possible that $\varepsilon_{\nu}(B)+\widetilde{V}^{\mathrm{MTF}}(u)=\mu$ on an open set of $u$ values. The minimizers are therefore not necessarily unique. The chemical potential $\mu$ is uniquely determined by (5.48) and the condition $(5.47)$.

We shall now prove that $\mu=\mu^{\mathrm{MTF}}$. All we have to show is that we can find functions $\rho_{\nu}$ satisfying (5.47) and (5.48) with $\mu=\mu^{\mathrm{MTF}}$.

We know from the MTF equation, Theorem 2.2, that if $\rho^{\mathrm{MTF}}(u)=0$ then $V^{\mathrm{MTF}}(u) \geq$ $\mu^{\mathrm{MTF}}$. Since $\widetilde{V}^{\mathrm{MTF}}$ differs from $V^{\mathrm{MTF}}$ only on the set $\rho^{\mathrm{MTF}}=0$ we may in $(5.48)$ when $\mu=\mu^{\mathrm{MTF}}$ replace $\widetilde{V}^{\mathrm{MTF}}$ by $V^{\mathrm{MTF}}$. We then know from the MTF equation that there are unique functions $\rho_{\nu}$ satisfying (5.48) with $\mu=\mu^{\mathrm{MTF}}$ and $\sum_{\nu} \rho_{\nu}(u)=\rho^{\mathrm{MTF}}(u)$. In fact, in 
terms of the filling factors (5.17) we have $\rho_{\nu}(u)=f_{\nu}(u) d_{\nu}(B)$. Since $\int \rho^{\mathrm{MTF}}(u) \mathrm{d} u=N$ we have produced the functions $\rho_{\nu}$ allowing us to conclude that $\mu$ is indeed equal to $\mu^{\mathrm{MTF}}$. If we insert these functions in $(5.45)$ we obtain

$\sum_{\nu} \int \varepsilon_{\nu}(B) \rho_{\nu}(u) \mathrm{d} u+\int V^{\mathrm{MTF}}(u) \rho^{\mathrm{MTF}}(u) \mathrm{d} u=\int\left(j_{B}\left(\rho^{\mathrm{MTF}}(u)\right)+V^{\mathrm{MTF}}(u) \rho^{\mathrm{MTF}}(u)\right) \mathrm{d} u$,

where the identity follows from (2.3).

We can now combine $(5.38),(5.39),(5.43),(5.44)$ and (5.49) to arrive at

$$
\begin{aligned}
E^{\mathrm{Q}}(N, B, V) & \geq(1-\varepsilon) E^{\mathrm{MTF}}(N, B, V)-\varepsilon D\left(\rho^{\mathrm{MTF}}, \rho^{\mathrm{MTF}}\right) \\
& -(\text { const. })\left(\sqrt{\beta_{c}} N^{3 / 2}+\varepsilon^{-1} N\right)-C(v) N^{5 / 3}
\end{aligned}
$$

Hence, since $D\left(\rho^{\mathrm{MTF}}, \rho^{\mathrm{MTF}}\right) \leq E^{\mathrm{MTF}}(N, B, V)$ we have

$$
\begin{aligned}
N^{-2}\left(E^{\mathrm{Q}}(N, B, V)-E^{\mathrm{MTF}}(N, B, V)\right) & \geq-2 \varepsilon E^{\mathrm{MTF}}(1, B / N, v) \\
& -(\text { const. })\left(\sqrt{\beta_{c}} N^{-1 / 2}+\varepsilon^{-1} N^{-1}+c(v) N^{-1 / 3}\right) .
\end{aligned}
$$

Note that $E^{\mathrm{MTF}}(1, B / N, v)$ is bounded by a constant depending only on $v$. If we choose $\varepsilon \sim N^{-1 / 2}$ we find

$$
N^{-2}\left(E^{\mathrm{Q}}(N, B, V)-E^{\mathrm{MTF}}(N, B, V) \geq-c^{-}(v)\left[\sqrt{\beta_{c}} N^{-1 / 2}+N^{-1 / 3}\right] .\right.
$$

This is equivalent to $(5.27)$ with $\varepsilon_{N}^{-}(v)=c^{-}(v)\left[\sqrt{\beta_{c}} N^{-1 / 2}+N^{-1 / 3}\right]$. In the case when $\rho^{\mathrm{C}}$ is bounded $\beta_{c}$ is a constant, otherwise we chose it to be $\beta_{c}=N^{1 / 3}$. In both cases will $\varepsilon_{N}^{-}(v)$ tend to zero as $N$ tends to infinity. This finishes the proof of the lower bound.

We have proved (1.7). The limits in (1.8) and (1.9) follow immediately from the corresponding results for $E^{\mathrm{MTF}}$ proved in Sects. II and III.

\subsection{HOMOGENEOUS EXTERIOR POTENTIALS}


Finally, we shall show how to prove the stronger result Theorem 1.3 for homogeneous exterior potentials.

In this case we do not have that the minimizing MTF density is supported within a fixed ball. In fact, the density will spread out as the coupling constant becomes small.

We shall prove that given $\varepsilon>0$ and $k_{0}$ there is an $N_{\varepsilon}$ independent of $B$ such that for $N \geq N_{\varepsilon}$ and $K / N \leq k_{0}$

$$
\left|E^{\mathrm{Q}}(N, B, K v) / E^{\mathrm{MTF}}(N, B, K v)-1\right|<\varepsilon .
$$

We consider large and small $K$ in very much the same way as we did for $B$ in the lower bound above. We shall see below that we can find a $k_{c}$ (depending only on $v$ and $\varepsilon$ ) such that (5.51) holds for $K / N \leq k_{c}$.

In the case of large $K$, i.e., $K / N \geq k_{c}$ (but $K / N \leq k_{0}$ ) the proof of (5.51) is then identical to the proof of Theorem 1.1 given above.

For small $K$ we again consider the upper and lower bounds separately. We begin with the upper bound. We proceed as in Sect. 5.1. We define the trial operator as in (5.17) and (5.18) except that we replace $\rho^{\mathrm{MTF}}$ by $\rho_{N, K v}^{\mathrm{C}}$. The estimate (5.20) now becomes $E^{\mathrm{Q}}(N, B, K v) \leq \mathcal{E}^{\mathrm{MTF}}\left[\rho_{N, K v}^{\mathrm{C}} ; B, K v\right]+N r^{-2} \int(\nabla g(x))^{2} \mathrm{~d} x+N K \sup _{|x| \leq R_{K}}\left[v * g_{r}^{2}(x)-v(x)\right]$ Here $R_{k}$ is the radius of the ball containing the support of $\rho_{N, K V}^{\mathrm{C}}$ for $K=k N$. According to $(3.22) R_{k}=k^{-1 /(s+1)} R_{1}$, where $R_{1}$ is the radius for $k=1$, which depends only on $v$.

Using the homogeneity of $v$ we have

$$
v * g_{r}^{2}(x)-v(x)=|x|^{s} \int[v((x-y) /|x|)-v(x /|x|)] g_{r}^{2}(y) \mathrm{d} y \leq c_{3}(v) r|x|^{s-1}
$$


Thus from (5c.1) we obtain since $s>1$

$$
\begin{aligned}
E^{\mathrm{Q}}(N, B, K v)-\mathcal{E}^{\mathrm{MTF}}\left[\rho^{\mathrm{C}} ; B, K v\right] & \leq c_{4}(v) N\left[r^{-2}+K r R^{s-1}\right] \\
& \leq c_{5}(v) N\left[r^{-2}+K r(K / N)^{-(s-1) /(s+1)}\right] \\
& \leq c_{5}(v) N^{2 / 3}(K / N)^{4 /(3(s+1))},
\end{aligned}
$$

with the choice $r=(K / N)^{-2 /(3(s+1))}$. We also know from (3.25) that

$$
\mathcal{E}^{\mathrm{MTF}}\left[\rho^{\mathrm{C}} ; B, K v\right] \leq(K / N)^{2 /(s+1)} N^{2} \int j_{0}\left(\rho_{1, v}^{\mathrm{C}}\right)+E^{\mathrm{C}}(N, K v)
$$

Hence, from (3.21) and (3.17) we obtain

$$
\begin{aligned}
E^{\mathrm{Q}}(N, B, K v) & \leq\left(1+\frac{c_{6}(v) N^{2}}{E^{\mathrm{C}}(N, K v)}\left[N^{-1 / 3}(K / N)^{4 /(3(s+1))}+(K / N)^{2 /(s+1)}\right]\right) E^{\mathrm{C}}(N, K v) \\
& \leq\left(1-c_{7}(v)\left[N^{-1 / 3}(K / N)^{1 /(3(s+1))}+(K / N)^{1 /(s+1)}\right]\right) E^{\mathrm{C}}(N, K v)
\end{aligned}
$$

(recall that $E^{\mathrm{C}}(N, K v)$ is negative, hence the minus sign in the second line). It therefore follows from Theorem 3.5 that we can find $k_{c}$ depending only on $\varepsilon$ (but not on $B$ ) such that $E^{\mathrm{Q}}(N, B, K v) / E^{\mathrm{MTF}}(N, B, K v) \geq 1-\varepsilon$ for $K / N \leq k_{c}$.

We turn next to the lower bound. As in Sect. 5.5 (in the case $B / N \geq \beta_{c}$ ) we may ignore the kinetic energy operator, which we had normalized to be positive. We then have the obvious inequality $E^{\mathrm{Q}}(N, B, V) \geq E^{\mathrm{P}}(N, V)$ where $E^{\mathrm{P}}$ is the energy of the classical point problem.

We shall use Lemma 4.3 to compare $E^{\mathrm{P}}(N, K v)$ to $E^{\mathrm{C}}(N, K v)$. We must, however, first discuss the scaling of $E^{\mathrm{P}}(N, K v)$. It is clear that if $v$ is homogeneous of degree $s$ then

$$
\mathcal{E}^{\mathrm{P}}\left(x_{1}, \ldots, x_{1} ; K v\right)=k^{1 /(s+1)} \mathcal{E}^{\mathrm{P}}\left(k^{1 /(s+1)} x_{1}, \ldots, k^{1 /(s+1)} x_{1} ; v\right) .
$$

Therefore, $E^{\mathrm{P}}(N, k V)=k^{1 /(s+1)} E^{\mathrm{P}}(N, V)$, i.e., $E^{\mathrm{P}}(N, k V)$ has the same scaling as $E^{\mathrm{C}}(N, K v)$ $[$ see $(3.21)]$. 
From Lemma 4.3 we thus find that

$$
E^{\mathrm{P}}(N, K v) \geq E^{\mathrm{C}}(N, K v)-b(v)(K / N)^{1 /(s+1)} N^{3 / 2} \geq E^{\mathrm{C}}(N, K v)\left(1+c_{8}(v) N^{-1 / 2}\right)
$$

According to Theorem 3.5 we may thus assume that $k_{c}$ is such that

$$
E^{\mathrm{Q}}(N, B, K v) / E^{\mathrm{MTF}}(N, B, K v) \leq(1+\varepsilon / 2)\left(1+c_{8}(v) N^{-1 / 2}\right)
$$

for $K / N \leq k_{c}$. We can therefore clearly find $N_{\varepsilon}$ such that the right side of (5.53) is less than $1+\varepsilon$ for $N \geq N_{\varepsilon}$.

\section{CONCLUSION}

We have analyzed the ground state of a two-dimensional gas of $N$ electrons interacting with each other via the (three-dimensional) Coulomb potential and subject to a confining exterior potential $V(x)=K v(x)$ where $K$ is an adjustable coupling constant. The electrons are also subject to a uniform magnetic field $B$ perpendicular to the two dimensional plane.

We have found the exact energy and electron density function $\rho(x)$ to leading order in $1 / N$, i.e., in the high density limit. This limit is achieved by letting $K$ be proportional to $N$ as $N \rightarrow \infty$, thus effectively confining the electrons to a fixed region of space, independent of $N$.

It turns out that the answer to the problem depends critically on the behavior of $B$ as $N \rightarrow \infty$. There are three regimes.

(i) If $B / N \rightarrow 0$, i.e., $N \gg B$ in appropriate units, then normal (two-dimensional) Thomas-Fermi theory gives the exact description. Correlations can be ignored to leading order in this high density situation.

(ii) If $B / N=$ constant, a modified TF theory in which the "kinetic energy density" is changed from (const.) $\rho^{2}$ to a certain $B$-dependent function of $\rho\left(\right.$ called $\left.j_{B}(\rho)\right)$ is exact. 
(iii) If $B / N \rightarrow \infty$ then the kinetic energy term can be omitted entirely and a classical continuum electrostatics theory emerges as the exact theory. This electrostatics problem is mathematically interesting in its own right and can be solved in closed form for the customary choice $v(x)=|x|^{2}$.

Related to the continuum problem is an electrostatics problem for point charged particles. Apart from its mathematical interest, it provides a crucial lower bound to the energy in case (iii). Another technical point of some interest is the extension of the Lieb-Thirring inequality to the two-dimensional particles in a magnetic field which involves dealing with a continuum of zero energy modes (i.e., the lowest Landau level).

\section{APPENDIX}

Here we prove that the minimizers for our three semi-classical problems can be sought among densities that vanish outside some finite radius — for which we give an upper bound. This lemma is in an appendix because it pertains to several sections of the paper.

A.1. LEMMA (Finite radius of minimizers). Consider the three cases: (a) The classical energy; (b) the classical particle energy; (c) the MTF energy. Let $V(x)$ be the confining potential. We assume that $V(x) \rightarrow+\infty$ as $|x| \rightarrow \infty$ in the sense that the number $W(R):=\inf \{V(x):|x| \geq R\}$ tends to $\infty$ as $R \rightarrow \infty$.

Then there is a radius $R_{v}$, depending only on $v=V / N$

$$
\begin{aligned}
E^{\mathrm{P}}(N, V) & =\inf \left\{\mathcal{E}^{\mathrm{P}}\left(x_{1}, \ldots, x_{N}\right):\left|x_{i}\right| \leq R_{v} \text { for all } i\right\} \\
E^{\mathrm{C}}(N, V) & =\inf \left\{\mathcal{E}^{\mathrm{C}}[\mu ; V]: \text { support } \mu \subset\left\{x:|x| \leq R_{v}\right\}, \int d \mu=N\right\} \\
E^{\mathrm{MTF}}(N, B, V) & =\inf \left\{\mathcal{E}^{\mathrm{MTF}}[\rho ; B, V]: \rho(x)=0 \text { for }|x|>R_{v}, \int \rho=N\right\} .
\end{aligned}
$$

Furthermore, any minimizing particle distribution measure or density satisfies the conditions given in braces in (a.1) 
A choice for $R_{v}$, which is far from optimal, is any $R$ satisfying the inequality

$$
\frac{1}{N} W(R) \geq\left(2+\pi^{-1}\right)+\frac{1}{N}\langle V\rangle_{1},
$$

with $\langle V\rangle_{1}$ being the average of $V$ in the unit disc:

$$
\langle V\rangle_{1}=\frac{1}{\pi} \int_{|x|<1} V(x) d x
$$

Proof: Particle case: Suppose that $\left|x_{1}\right|>R_{v}$. Then we move particle 1 inside $D$, the unit disc centered at the origin. The point, $y$, to which we move particle 1 is not known, so we average the energy over all choices of $y \in D$. If we show that this average energy is less than the original energy then we know that there is some point $y \in D$ such that the energy is lowered. Thus, we have to show that

$$
V\left(x_{1}\right)+\sum_{j=2}^{N}\left|x_{1}-x_{j}\right|^{-1}>\langle V\rangle_{1}+\frac{1}{\pi} \sum_{j=2}^{N} \int_{D}\left|y-x_{j}\right|^{-1} d y .
$$

Noting that $\int_{D}|y-x|^{-1} d y \leq \int_{D}|y|^{-1} d y=2 \pi$, by a simple rearrangement inequality, we see that it suffices to have $W\left(R_{v}\right)>\langle V\rangle_{1}+2 N$, which agrees with (a.2).

The Classical case: If $\mu$ is any measure with $\int d \mu=N$, we define $\mu^{+}$to be $\mu$ restricted to the complement of the closed disc of radius $R_{v}$ centered at the origin. Thus $\mu^{+}(A)=$ $\mu\left(A \cap\left\{x:|x|>R_{v}\right\}\right)$. Similarly, $\mu^{-}$is $\mu$ restricted to the disc, so that $\mu=\mu^{+}+\mu^{-}$. Assuming that

$\mu^{+} \neq 0$, we replace $\mu$ by $\mu_{\varepsilon}:=(1-\varepsilon) \mu^{+}+\mu^{-}+\delta \nu$, where $\nu$ is Lebesgue measure restricted to the unit disc, $D$, and where $\pi \delta=\varepsilon \int d \mu^{+}$. Thus $\int d \mu_{\varepsilon}=N$. The change in energy, to $O(\varepsilon)$ as $\varepsilon \downarrow 0$, is easily seen to be

$\delta \int_{D} V(x) d x-\varepsilon \int V(x) \mu^{+}(d x)+\delta \int_{D} \int_{\mathbf{R}^{2}}|x-y|^{-1} d x \mu(d y)-\varepsilon \iint|x-y|^{-1} \mu^{+}(d x) \mu(d y)$ 
$<\pi \delta\langle V\rangle_{1}-\varepsilon W\left(R_{v}\right) \int d \mu^{+}+2 \pi \delta N$, which is negative by (a.1).

The MTF case: This is similar to the classical case, but with two differences: (i) The measure $\mu$ is replaced by a function $\rho$ with $\int \rho(x) d x=N$ and (ii) a "kinetic energy" term $\int j_{B}(\rho(x)) d x$ is added to the energy. Point (i) only simplifies matters. For point (ii) we note the simple fact that $j_{B}(\rho)$ is bounded above by $\pi \rho^{2} / 2$ and its derivative, $j^{\prime}(\rho)$ is bounded above by $\pi \rho$; this is true for all $B$. Let us assume that $d \mu^{+}:=\rho^{+}(x) d x$ is not zero, with $\rho^{+}(x)=\rho(x)$ for $|x|>R_{v}$ and $\rho^{+}(x)=0$ otherwise. The argument is as before, but now we must take into account the change in kinetic energy which, to leading order in $\varepsilon$, is

$$
\delta \int_{D} j_{B}^{\prime}(\rho(x)) d x-\varepsilon \int_{B}^{\prime}\left(\rho^{+}(x)\right) d x<\delta \pi \int_{D} \rho(x) d x \leq \delta N=\varepsilon \int \rho^{+}(x) d x .
$$

The total energy change is then negative by (a.1).

Q.E.D.

Acknowledgements We thank Vidar Gudmundsson and Jari Kinaret for valuable discussions and Kristinn Johnsen for allowing us to present the picture of electronic densities in quantum dots that he prepared. 


\section{REFERENCES}

[1] H. van Houten, C.W.J. Beenakker, and A.A.M. Staring, in: Single Charge Tunneling, eds. H. Grabert, J.M. Martinis and M.H. Devoret, Plenum, New York, (1991).

[2] M.A. Kastner, The single electron transistor, Rev. Mod. Phys. 64, 849-859 (1992)

[3] P.L. McEuen, E.B. Foxman, J. Kinaret, U. Meirav, M.A. Kastner, N.S. Wingreen and S.J. Wind, Self consistent addition spectrum of a Coulomb island in the quantum Hall regime, Phys. Rev. B 45, 11419-11422 (1992).

[4] R.C. Ashoori, H.L. Stormer, J.S. Weiner, L.N. Pfeiffer, S.J. Pearton, K.W. Baldwin, and K.W. West Single-Electron Capacitance Spectroscopy of Discrete Quantum Levels, Phys. Rev. Lett. 68, 3088-3091 (1992)

[5] R.C. Ashoori, H.L. Stormer, J.S. Weiner, L.N. Pfeiffer, K.W. Baldwin, and K.W. West N-electron Ground State Energies of a Quantum Dot in Magnetic Field, Phys. Rev. Lett. 71, 613-616 (1993)

[6] N.C. van der Vaart, M.P. de Ruyter van Steveninck, L.P. Kouwenhoven, A.T. Johnson, Y.V. Nazarov, and C.J.P.M. Harmans, Time-Resolved Tunneling of Single Electrons between Landau Levels in a Quantum Dot, Phys. Rev. Lett. 73, 320-323 (1994)

[7] O. Klein, C. Chamon, D. Tang, D.M. Abush-Magder, X.-G. Wen, M.A. Kastner, and S.J. Wind, Conductance of an Artificial Atom in Strong Magnetic Fields, preprint, (1994)

[8] A. Kumar, S.E. Laux and F. Stern, Electron states in a GaAs quantum dot in a magnetic field, Phys. Rev. B. 42, 5166-5175 (1990).

[9] C.W.J. Beenakker, Theory of Coulomb-blockade oscillations in the conductance of a quantum dot, Phys. Rev. B 44, 1646-1656 (1991)

[10] V. Shikin, S. Nazin, D. Heitmann, and T. Demel, Dynamic response of quantum dots, Phys. Rev. B, 43, 11903-11907 (1991). 
[11] V. Gudmundsson, R.R. Gerhardts, Self-consistent model of magnetoplasmons in quantum dots with nearly parabolic confinement potentials, Phys. Rev. B 43, 12098-12101 (1991)

[12] A.H. MacDonald, S.R. Eric Yang, and M.D. Johnson, Quantum Dots in Strong Magnetic Fields: Stability Criteria for the Maximum Density Droplet, Aust. J. Phys. 46, $345-58(1993)$

[13] P.L. McEuen, N.S. Wingreen, E.B. Foxman, J. Kinaret, U. Meirav, M.A. Kastner, and S.J. Wind, Coulomb interactions and energy-level spectrum of a small electron gas, Physica B 189, 70-79 (1993).

[14] S.-R. Eric Yang, A.H. MacDonald, and M.D. Johnson, Addition Spectra of Quantum Dots in Strong Magnetic Fields, Phys.Rev. Letters 71, 3194-3197 (1993)

[15] J.M. Kinaret and N.S. Windgreen, Coulomb blockade and partially transparent tunneling barriers in the quantum Hall regime, Phys. Rev. B, 48, 11113-11119 (1993)

[16] D. Pfannkuche, V. Gudmundsson, P.A. Maksym, Comparison of a Hartree, a HartreeFock, and an exact treatment of quantum dot helium, Phys. Rev. B, 47, 2244-2250 (1993).

[17] D. Pfannkuche, V. Gudmundsson, P. Hawrylak, and R.R. Gerhardts, Far-Infrared Response of Quantum Dots: From Few Electron Exitations to Magnetoplasmons, Solid State Electronics 37, 1221-1226 (1994)

[18] M. Ferconi and G. Vignale, Current density functional theory of quantum dots in a magnetic field, preprint (1994)

[19] E.H. Lieb, J.P. Solovej and J. Yngvason, Heavy Atoms in the Strong Magnetic Field of a Neutron Star, Phys. Rev. Lett. 69, 749-752 (1992). 
[20] E.H. Lieb, J. P. Solovej and J. Yngvason, Asymptotics of Heavy atoms in High magnetic Fields: I. Lowest Landau Band Regions, Commun. Pure. Appl. Math. 47, 513-591 (1994)

[21] E.H. Lieb, J. P. Solovej and J. Yngvason, Asymptotics of Heavy atoms in High magnetic Fields: II. Semiclassical Regions, Commun. Math. Phys 161, 77-124 (1994)

[22] E. H. Lieb, J.P. Solovej and J. Yngvason, Quantum Dots, in: Proceedings of the conference on partial differential equations and mathematical physics, Birmingham, Alabama, 1994, International Press (in press)

[23] Tomishima and K. Yonei, Thomas-Fermi Theory for Atoms in a Strong Magnetic Field, Progr. Theor. Phys. 59, 683-696 (1978).

[24] I. Fushiki, E.H. Gudmundsson, C. J. Pethick, and J. Yngvason, Matter in a Magnetic Field in the Thomas-Fermi and Related Theories, Ann. Phys. 216, 29-72 (1992).

[25] J. Yngvason, Thomas-Fermi Theory for Matter in a Magnetic Field as a Limit of Quantum Mechanics, Lett. Math. Phys. 22, 107-117 (1991).

[26] E.H. Lieb and H.-T. Yau, The stability and instability of relativistic matter, Commun. Math. Phys. 118, 177-213 (1988).

[27] V. Fock, Bemerkung zur Quantelung des harmonischen Oszillators in Magnetfeld, Z. Phys. 47, 446-448 (1928).

[28] E.H. Lieb, Thomas-Fermi and related theories of atoms and molecules, Rev. Mod. Phys. 53, 603-641 (1981); Erratum, Rev. Mod. Phys. 54, 311 (1982).

[29] E.H. Lieb and B. Simon, The Thomas-Fermi Theory of Atoms, Molecules and Solids, Adv. in Math. 23, 22-116 (1977).

[30] E.H. Lieb and M. Loss, unpublished section of a book on stability of matter.

[31] E.H. Lieb and W. E. Thirring, Bound for the Kinetic Energy of Fermions Which Proves the Stability of Matter, Phys. Rev. Lett, 35, 687-689 (1975). 
[32] E.H. Lieb and W.E. Thirring, Inequalities for the moments of the eigenvalues of the Schrödinger Hamiltonian and their relation to Sobolev inequalities, in: Studies in Mathematical Physics: Essays in Honor of Valentine Bargmann (E.H. Lieb, B. Simon and A. Wightman eds.), 269-303, Princeton University Press, 1976.

[33] L. Erdős, Magnetic Lieb-Thirring inequalities, to appear in Comm. Math. Phys.

[34] E.H. Lieb, A lower bound for Coulomb energies, Phys. Lett. 70A, 444-446 (1979).

[35] E.H. Lieb and S. Oxford, An Improved Lower Bound on the Indirect Coulomb Energy, Int. J. Quant. Chem. 19, 427-439 (1981).

[36] E.H. Lieb, J. P. Solovej, Quantum coherent operators: A generalization of coherent states, Lett. Math. Phys. 22, 145-154 (1991).

[37] E.H. Lieb, A Variational Principle for Many-Fermion Systems, Phys. Rev. Lett. 46, 457-459; Erratum 47, 69 (1981).

[38] B. Simon, Functional Integration and Quantum Physics, Academic Press, 1979

[39] K. Fan, Maximum Properties and Inequalities for the Eigenvalues of Completely Continuous Operators, Proc. Nat. Acad. Sci. 37 (1951)

[40] E.H. Lieb, On Characteristic Exponents in Turbulence, Commun. Math. Phys. 92, 57-121 (1984).

[41] Ph. Blanchard and J. Stubbe, Phase space bounds for Schrödinger Hamiltonians and applications, Preprint.

[42] A. Martin, New results on the moments of the eigenvalues of the Schrödinger Hamiltonian and applications, Commun. Math. Phys. 129, 161-168 (1990)

[43] E.H. Lieb, Kinetic Energy Bounds and their Application to the Stability of Matter, in: Schrödinger Operators, Proceedings of a conference in Sønderborg Denmark 1988, H. Holden and A. Jensen eds., Springer Lecture Notes in Physics 345, 371-382 (1989). 
[44] V. Bach, Error bound for the Hartree-Fock energy of atoms and molecules, Commun. Math. Phys. 147, 527-548 (1992).

[45] C. Fefferman and R. de la Llave, Relativistic stability of matter. I., Rev. Iberoamericana 2, 119-215 (1986).

[46] E.M. Stein and G. Weiss, Fourier analysis on Euclidean spaces, Princeton University Press, 1971. 